\title{
Numerical Analysis of Multidomain Systems: Coupled Nonlinear PDEs and DAEs With Noise
}

\author{
Alper Demir ${ }^{(}$, Fellow, IEEE, and M. Selim Hanay
}

\begin{abstract}
We present a numerical modeling and simulation paradigm for multidomain, multiphysics systems with components modeled both in a lumped and distributed manner. The lumped components are modeled with a system of differential-algebraic equations (DAEs), whereas the possibly nonlinear distributed components that may belong to different physical domains are modeled using partial differential equations (PDEs) with associated boundary conditions. We address a comprehensive suite of problems for nonlinear coupled DAE-PDE systems including 1) transient simulation; 2) periodic steady-state (PSS) analysis formulated as a mixed boundary value problem that is solved with a hierarchical spectral collocation technique based on a joint Fourier-Chebyshev representation, for both forced and autonomous systems; 3) Floquet theory and analysis for coupled linear periodically time-varying DAE-PDE systems; 4) phase noise analysis for multidomain oscillators; and 5) efficient parameter sweeps for PSS and noise analyses based on first-order and pseudo-arclength continuation schemes. All of these techniques, implemented in a prototype simulator, are applied to a substantial case study: a multidomain feedback oscillator composed of distributed and lumped components in two physical domains, namely, a nano-mechanical beam resonator operating in the nonlinear regime, an electrical delay line, an electronic amplifier and a sensor-actuator for the transduction between the two physical domains.
\end{abstract}

Index Terms-Chebyshev and Fourier representations and collocation, differential-algebraic equations (DAEs), mixed boundary value problems, multidomain systems, multiphysics simulation, nano electro-mechanical systems (NEMS), noise, oscillators, partial differential equations (PDEs), phase noise, spectral methods.

\section{INTRODUCTION}

$\mathbf{I}$ $\mathrm{N}$ THE design and analysis of engineering systems, one often has to take into account interaction between different physical phenomena, and perform a so-called multiphysics analysis [1]. For instance, the dynamics of electronic circuits are governed by the laws of electromagnetism as encapsulated by Maxwell's equations or Kirchoff's laws. On the other hand, the reliability and performance of integrated circuits critically depend on the local operation temperature [2]. In integrated

Manuscript received October 12, 2016; revised March 3, 2017 and May 22, 2017; accepted September 9, 2017. Date of publication September 18, 2017; date of current version June 18, 2018. This paper was recommended by Associate Editor A. Raychowdhury. (Corresponding author: Alper Demir.)

A. Demir is with the Department of Electrical and Electronics Engineering, Koç University, Istanbul 34450, Turkey (e-mail: aldemir@ku.edu.tr).

M. S. Hanay is with the Department of Mechanical Engineering, Bilkent University, Ankara 06800, Turkey.

Color versions of one or more of the figures in this paper are available online at http://ieeexplore.ieee.org.

Digital Object Identifier 10.1109/TCAD.2017.2753699 circuit design, one has to carefully evaluate the impact of heat generation and thermal effects and, if necessary, introduce effective cooling solutions. This involves performing a coupled multiphysics analysis of both the electrical dynamics and the thermal behavior of the circuit.

The interaction of multiple physical phenomena is often undesired and results in degraded system performance. On the other hand, there are many interesting and emerging engineering systems that have a multidomain nature. For instance, nano electro-mechanical systems (NEMS) contain tightly interacting and integrated mechanical and electronic components exhibiting multiple and coupled physical phenomena, governed by the laws of mechanics, electromagnetism, optics, piezo-resistivity, etc. [3]. The utility and novelty of these kinds of systems critically stem from their multidomain nature. The possibility of putting together different domains in an engineering system is opening up vast opportunities and enabling unprecedented applications. However, the design and analysis of such multidomain systems pose a huge challenge. The electronic design automation community is already grappling with very difficult problems in the design and analysis of purely electronic systems, due to shrinking device sizes and ever increasing system complexity. The multidomain nature of the emerging systems that bring together electronics with other domains turns an already difficult problem into an extreme challenge that needs to be addressed.

There are various software packages for simulating multiphysics models [1], which are mostly based on solving a coupled system of partial differential equations (PDEs), mainly employing finite element methods for numerical computations. This approach is no doubt very general. However, it is also very costly to model every component of a system with a PDE in a distributed manner. As a result, only basic types of analyses, e.g., dc/static, ac, and transient, can be conducted for relatively small scale systems. On the other hand, various types of detailed and advanced analyses, such as periodic steady-state (PSS) and nonstationary noise, can be conducted at the electrical level for large electronic circuits using mostly lumped component models encapsulated as a system of differential-algebraic equations (DAEs). In fact, lumped models would be adequate for most components of multidomain systems, with only a few requiring a judiciously chosen distributed, PDE-based high fidelity model. We thus propose a numerical modeling and simulation paradigm for multidomain, multiphysics systems with components modeled both in a distributed and lumped manner. The lumped components are modeled with a system of DAEs, whereas the 
distributed components that may belong to different physical domains are modeled using PDEs with associated boundary conditions (BCs).

We envision that electronic circuit simulators that are traditionally based on a DAE formulation can be enhanced and extended to a coupled DAE-PDE formalism. The state-of-theart circuit simulators already contain models that can handle some specialized distributed components. However, these are linear models mostly for interconnect structures. In current practice, one has to first develop or derive a DAE compatible, lumped behavioral model for the nonlinear distributed components, possibly using other tools, in order to include them in the circuit simulator. In this paper, our eventual goal is to augment circuit simulators so that they can handle various kinds of nonlinear, as well as linear, distributed components in a transparent and painless manner. It is most desirable that the rich suite of analysis schemes (steady-state, noise, etc.) already available in a circuit simulator be extended for nonlinear coupled DAE-PDE systems. This nontrivial and challenging task requires rethinking many aspects of a circuit simulator and the development of novel formulations and algorithms, e.g., a new circuit and system specification scheme, new formulations for various analysis types and the extension of the theory behind them, and new numerical methods.

We address a comprehensive suite of problems for the numerical modeling and analysis of multidomain systems modeled as nonlinear coupled DAE-PDE systems. In Section II, we first set up a general formulation that captures coupled DAE-PDE systems. Then in Section III, we present a substantial case study: a multidomain feedback oscillator composed of distributed and lumped components in two physical domains, namely, a nano-mechanical beam resonator operating in the nonlinear regime, an electrical delay line, an electronic amplifier, and a sensor-actuator for the transduction between the two physical domains. This example serves as a concretization for the abstract formulations we present. It is used throughout this paper in order to illustrate the techniques we discuss. In Section IV, we discuss the linearization of nonlinear coupled DAE-PDE systems at the functionaloperator level, which significantly differs from that of pure DAE systems. These linearizations form the basis for various analysis schemes and numerical methods that will be discussed later. In Section V, we present a generalization of Floquet theory to coupled DAE-PDE systems. This serves as the basis for phase noise analysis of multidomain oscillators, that we discuss in Section VI. Then in Section VII, we describe the numerical techniques we have formulated and implemented in a prototype simulator for multidomain systems. In particular, we discuss novel formulations and techniques for PSS analysis, Floquet analysis and phase noise analysis. Some aspects of the theory, analysis formulations and the numerical techniques are inherited from the pure DAE formulations in circuit simulators and the numerical methods recently developed by the numerical analysis community. However, the handling of the PDE-based models for the nonlinear distributed components coupled with the DAE-based lumped models, and the formulation of PSS, Floquet and phase noise analyses for such systems, require nontrivial extensions. In particular, we present a new hierarchical spectral collocation scheme based on a joint Fourier-Chebyshev representation for PSS analysis that also forms the basis for the subsequent Floquet and phase noise analyses. We believe that spectral methods, as opposed to finite element schemes, are more appropriate for handling the distributed components, due to their superior efficiency and accuracy [4]. They are also more compatible with the numerical techniques already in use for circuit simulation. Finally, in Section VIII, we present numerical analysis results for our case study, the mechanical beam resonator and the NEMS oscillator. We compare our noise analysis results with the ones from recent literature that were obtained in a semi-analytical manner based on a purely lumped model. The use of distributed models for key components of a multidomain system can offer better design insight and improved analysis fidelity.

The main novel contributions of this paper are as follows.

1) Conceptualization of nonlinear coupled DAE-PDEbased simulation paradigm for multiphysics simulation of multidomain systems in a circuit simulator.

2) Hierarchical, joint Fourier-Chebyshev spectral collocation scheme for PSS analysis of nonlinear multidomain forced and autonomous systems modeled with coupled DAEs-PDEs.

3) Numerical Floquet and phase noise analysis scheme for multidomain oscillators as coupled DAE-PDE systems.

4) Practically relevant results on a substantial case study, i.e., a multidomain feedback oscillator used in attogram mass sensing applications, with comparisons to results in the literature.

\section{Coupled Multidomain PDEs And DAEs}

Let $t$ denote time and consider a multidomain, multiphysics dynamical system with distributed and lumped components. Let $\mathbf{x}=\left[x_{1}, \ldots, x_{p}\right]^{T}$ denote a vector of space coordinates for the distributed components. One can have $p>3$, since the components of the system may belong to different physical domains. For instance, in an NEMS system, one can have both mechanical and electrical distributed components that require the use of independent coordinate systems. Let $\mathbf{u}(t, \mathbf{x})=\left[u_{1}(t, \mathbf{x}), \ldots, u_{n}(t, \mathbf{x})\right]^{T}$ denote a vector of $n$ distributed variables, i.e., a vector of functions (of $\mathbf{x}$ ) for a given $t$. Let $\mathbf{z}(t)=\left[z_{1}(t), \ldots, z_{m}(t)\right]^{T}$ be a vector of $m$ lumped variables, i.e., a vector of scalars for fixed $t$. The dynamics of the multiphysics/domain system is described by

$$
\frac{\partial}{\partial t} \mathcal{L}(\mathbf{u}, \mathbf{z})=\mathcal{R}(t, \mathbf{u}, \mathbf{z}) \quad \text { with ICs and BCs }
$$

where the left-hand-side (LHS) $\mathcal{L}$ and the right-hand-side (RHS) $\mathcal{R}$ denote a vector of integro-differential nonlinear operators and functionals, that operate on a vector of functions, i.e., $\mathbf{u}(t, \mathbf{x})$, and a vector of scalars, i.e., $\mathbf{z}(t)$. All of the components of the vector produced by the operators and functionals in $\mathcal{L}$ and $\mathcal{R}$ by acting on $\mathbf{u}(t, \mathbf{x})$ and $\mathbf{z}(t)$ are functions of time $t$. We next consider these components for a given $t$. For hybrid systems that involve both distributed and lumped variables, some of these components are functions (of $\mathbf{x}$ ), whereas others that correspond to the lumped components are scalars. We partition $\mathcal{L}(\mathbf{u}, \mathbf{z})$ and $\mathcal{R}(t, \mathbf{u}, \mathbf{z})$ as follows to reflect this structure:

$$
\mathcal{L}(\mathbf{u}, \mathbf{z})=\left[\begin{array}{c}
\mathcal{L}_{\mathbf{u}}(\mathbf{u}, \mathbf{z}) \\
\mathcal{L}_{\mathbf{z}}(\mathbf{u}, \mathbf{z})
\end{array}\right] \quad \mathcal{R}(t, \mathbf{u}, \mathbf{z})=\left[\begin{array}{c}
\mathcal{R}_{\mathbf{u}}(t, \mathbf{u}, \mathbf{z}) \\
\mathcal{R}_{\mathbf{z}}(t, \mathbf{u}, \mathbf{z})
\end{array}\right] .
$$




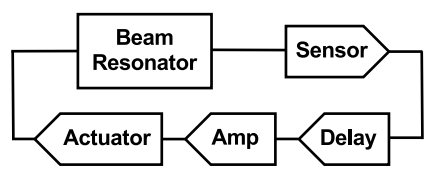

Fig. 1. NEMS oscillator with feedback configuration.

For a given $t$, the operators $\mathcal{L}_{\mathbf{u}}(\mathbf{u}, \mathbf{z})$ and $\mathcal{R}_{\mathbf{u}}(t, \mathbf{u}, \mathbf{z})$ (both with dimension $n \times 1)$ produce functions by acting on $\mathbf{u}(t, \mathbf{x})$ and $\mathbf{z}(t)$, whereas the functionals $\mathcal{L}_{\mathbf{z}}(\mathbf{u}, \mathbf{z})$ and $\mathcal{R}_{\mathbf{z}}(t, \mathbf{u}, \mathbf{z})$ (both with dimension $m \times 1$ ) produce scalars. The equation in (1) is associated with initial and/or BCs both in $t$ and $\mathbf{x}$. Without loss of generality, we assume that the derivatives with respect to time $t$ are restricted to be first-order. This is not a theoretical requirement, but is needed for the convenience of the numerical techniques that will be employed. If the governing equations contain higher-order derivatives with respect to $t$, auxiliary variables can be defined in order to transform the equations into the above form. Circuit simulators are based on a DAE formulation which is also restricted to have only firstorder time derivatives. On the other hand, there is no restriction on the order of derivatives with respect to $\mathbf{x}$ one may use in defining the operators $\mathcal{L}(\mathbf{u}, \mathbf{z})$ and $\mathcal{R}(t, \mathbf{u}, \mathbf{z})$. The RHS operator $\mathcal{R}(t, \mathbf{u}, \mathbf{z})$ is allowed to have an explicit dependence on $t$ in order to capture time-varying excitations. The formulation in (1) is most general, and was inspired by the pure DAE formulation in circuit simulators As a special case, it captures PDEs for distributed systems, DAEs for lumped systems, and hybrid models that contain both. For instance, modified nodal analysis (MNA) formulation of Kirchoff's laws for an electronic circuit, which is the basis of almost all circuit simulators, can be obtained from (1) if there are no distributed components, i.e., by removing $\mathbf{u}, \mathcal{L}_{\mathbf{u}}(\mathbf{u}, \mathbf{z})$ and $\mathcal{R}_{\mathbf{u}}(t, \mathbf{u}, \mathbf{z})$ from (2).

\section{Multidomain NEMS Oscillator}

In order to concretize the abstract formulation presented in Section II, we present a practically relevant case study, a multidomain NEMS oscillator with a block diagram given in Fig. 1. The NEMS oscillator is composed of a beam resonator, a sensor that converts the mechanical motion of the beam resonator into an electrical signal, an electrical delay line, an electronic amplifier, and an actuator that drives the beam resonator with the signal from the electronic amplifier [5], [6]. These components are connected in a feedback configuration and, under appropriate conditions, the system operates as an autonomous oscillator [7]. NEMS are currently being used as ultrasensitive mass and force sensors [8], [9].

\section{A. Mechanical Beam Resonator}

We model the mechanical beam resonator using the EulerBernoulli beam theory that applies to beams with length much larger than cross-sectional dimensions. The Euler-Bernoulli equation, a PDE, for a lossless and linear beam is given by

$$
m \frac{\partial^{2}}{\partial t^{2}} u(t, x)=-E I \frac{\partial^{4}}{\partial x^{4}} u(t, x)+a(t, x)
$$

where $m$ is the mass per unit length, $E$ is the Young's (elastic) modulus of the beam material, $I$ is the moment of inertia, ${ }^{1} x$ is the position coordinate along the length of the beam, and $u(t, x)$ represents the position-dependent lateral deflection, and $a(t, x)$ captures any excitation (in dimensions of force per unit length) [10], [11]. The dimensions for the quantities above are: 1) elastic modulus $E$ : force per unit area; 2) $m$ : mass per unit length; and 3) $I$ : fourth power of length. Thus, the $(\mathrm{R} / \mathrm{L}) \mathrm{HS}$ of (3) has a dimension of force per unit length. The Euler-Bernoulli equation in (3) must always be augmented with appropriate BCs. For instance, for a beam with both ends fixed and rigidly clamped [10], [11], we have ( $L$ : beam length)

$$
u(t, x)=0 \quad \text { and } \quad \frac{\partial}{\partial x} u(t, x)=0 \quad \text { at } \quad x=0, L .
$$

There are various fundamental loss mechanisms in mechanical resonators. For a lossy beam, the Euler-Bernoulli equation in (3) needs to be augmented as follows:

$$
\begin{array}{r}
\left(m \frac{\partial^{2}}{\partial t^{2}}+\frac{m \omega_{Q}}{Q_{V I}} \frac{\partial}{\partial t}+\frac{E I}{Q_{K V} \omega_{Q}} \frac{\partial}{\partial t} \frac{\partial^{4}}{\partial x^{4}}\right) u(t, x) \\
=-E I \frac{\partial^{4}}{\partial x^{4}} u(t, x)+a(t, x)
\end{array}
$$

where $Q_{V I}$ and $Q_{K V}$ are the quality factors of the resonator due to external (viscous damping) and internal material (Kelvin-Voigt damping) losses [12]. $\omega_{Q}$ is the frequency at which the quality factors are defined, and is chosen as the lowest vibration mode frequency of the linear-lossless resonator. The overall quality factor is $Q=1 /\left(1 / Q_{V I}+1 / Q_{K V}\right)$.

As the beam vibrates laterally, its length changes slightly. This creates tension in the beam, which is a nonlinear phenomenon. ${ }^{2}$ This tension effect is captured by further augmenting the Euler-Bernoulli equation as below [11]

$$
\begin{aligned}
\left(m \frac{\partial^{2}}{\partial t^{2}}+\right. & \left.\frac{m \omega_{Q}}{Q_{V I}} \frac{\partial}{\partial t}+\frac{E I}{Q_{K V} \omega_{Q}} \frac{\partial}{\partial t} \frac{\partial^{4}}{\partial x^{4}}\right) u(t, x) \\
& =\left(-E I \frac{\partial^{4}}{\partial x^{4}}+T_{e} \frac{\partial^{2}}{\partial x^{2}}\right) u(t, x)+a(t, x)
\end{aligned}
$$

where the tension $T_{e}$ is given by $T_{e}=A E \Delta L(t) / L$ in terms of the beam cross-sectional area $A$ and the elongation $\Delta L(t)$. Tension $T_{e}$ can be expressed as the result of the following nonlinear integro-differential functional acting on $u(t, x)$ :

$$
\mathcal{T}_{e}(\odot)=\frac{A E}{L}\left[\int_{0}^{L} \sqrt{\left(1+\frac{\partial}{\partial x} \odot\right)^{2}} d x-L\right] .
$$

The functional $\mathcal{T}_{e}(\odot)$ defined above operates on functions of $x$ and produces scalar values. The following relate the quantities above [10], [11]: 1) strain $=\Delta L(t) / L ; 2$ ) stress (force per unit area) $=E \Delta L(t) / L$; and 3) tension $=A \times$ stress.

\section{B. Electrical Delay Line}

We model the delay line with the advection equation [13]

$$
t_{d} \frac{\partial}{\partial t} g(t, x)=-\frac{\partial}{\partial x} g(t, x) \quad \text { with } \quad g(t, x=0)=s(t)
$$

\footnotetext{
${ }^{1}$ For rectangular cross-section: $I=w t^{3} / 12, w$ : width, $t$ : thickness [10].

${ }^{2}$ Intrinsic tension on the beam is assumed to be zero.
} 
where $x$ is the position along the delay line, $s(t)$ is the input signal to be delayed, and $t_{d}$ is the amount of desired delay. Equation (8) has the solution $g(t, x)=s\left(t-x t_{d}\right)$. If the line has unit length, then the signal at its termination is $g(t, x=1)=s\left(t-t_{d}\right)$, i.e., a delayed version of the signal at its input.

\section{Electronic Amplifier}

We use a lumped model for the electronic amplifier and formulate its governing equations using Kirchoff's laws in the MNA form as a system of DAEs as follows:

$$
\frac{d}{d t} \mathbf{q}(\mathbf{v}(t))+\mathbf{f}(\mathbf{v}(t), b(t))=\mathbf{0}
$$

where $\mathbf{v}(t)$ contains a vector of circuit variables, nonlinear function $\mathbf{q}(\cdot)$ captures the capacitive and inductive components, $\mathbf{f}(\cdot, \cdot)$ models the memoryless, resistive effects. $b(t)$ is the input and set to the output of the delay line as follows:

$$
b(t)=g(t, x=1)=s\left(t-t_{d}\right) .
$$

The output $y(t)$ of the amplifier is typically chosen as one of the voltage or current variables, selected by a vector $\mathbf{l}$ from the entries of $\mathbf{v}(t)$ with $y(t)=\mathbf{l}^{T} \mathbf{v}(t)$.

\section{Sensor and Actuator}

We use simple models for the transduction between the mechanical and electrical domains. We assume a sensor that measures the deflection of the beam at its mid-point, whose electrical output is fed to the input of the delay line. The beam resonator is driven by an actuator that applies a lateral, position-independent force that varies with $t$ as dictated by the output of the electronic amplifier. Thus, the sensor and the actuator are represented by

$$
s(t)=u(t, x=L / 2), \quad a(t, x)=y(t)=\mathbf{l}^{T} \mathbf{v}(t) .
$$

\section{E. Unified Model}

We now put together the models of the components of the NEMS oscillator and obtain a unified model expressed in the general form in (1). We first set up a unified variable naming convention that is compatible with (1). We observe that the system model has distributed variables in two different physical domains, i.e., the lateral deflection $u(t, x)$ of the mechanical resonator, and the electrical signal $g(t, x)$ in the delay line. The space coordinates $x$ for these distributed variables are independent and belong to different domains: we set $\mathbf{x}=\left[x_{1}, x_{2}\right]^{T}$ with $x_{1}$ for the mechanical domain and $x_{2}$ for the electrical. We collect the distributed variables in $\mathbf{u}(t, \mathbf{x})=\left[u_{1}\left(t, x_{1}\right), u_{2}\left(t, x_{1}\right), u_{3}\left(t, x_{2}\right)\right]^{T}$ with $u_{1}\left(t, x_{1}\right)=$ $u\left(t, x_{1}\right)$ for the beam and $u_{3}\left(t, x_{2}\right)=g\left(t, x_{2}\right)$ for the delay line. $u_{2}\left(t, x_{1}\right)$ is reserved for the beam resonator, to be used shortly. The lumped variables of the system are contributed by only the amplifier: we set $\mathbf{z}(t)=\mathbf{v}(t)$.

We need to define auxiliary variables as necessary so that the DAEs and the PDEs that govern the system behavior can be formulated with only first-order derivatives with respect to time $t$

$$
u_{2}\left(t, x_{1}\right)=\frac{\partial}{\partial t} u_{1}\left(t, x_{1}\right)=\frac{\partial}{\partial t} u\left(t, x_{1}\right)
$$

and rewrite (6) as a set of two equations as follows:

$$
\begin{aligned}
& \frac{\partial}{\partial t} u_{1}\left(t, x_{1}\right)=u_{2}\left(t, x_{1}\right) \\
& \frac{\partial}{\partial t}\left(\frac{E I}{Q_{K V} \omega_{Q}} \frac{\partial^{4}}{\partial x_{1}^{4}} u_{1}\left(t, x_{1}\right)+m u_{2}\left(t, x_{1}\right)\right) \\
& \quad=\left(-E I \frac{\partial^{4}}{\partial x_{1}^{4}}+T_{e} \frac{\partial^{2}}{\partial x_{1}^{2}}\right) u_{1}\left(t, x_{1}\right)-\frac{m \omega_{Q}}{Q_{V I}} u_{2}\left(t, x_{1}\right)+a\left(t, x_{1}\right)
\end{aligned}
$$

with tension $T_{e}=\mathcal{T}_{e}\left(u_{1}\left(t, x_{1}\right)\right)$ defined by (7).

Finally, we put together all of the model equations above and obtain (15), as shown at the bottom of this page, with the BCs in (16), as shown at the bottom of this page. We note that (15) is in the form given in (1). Operators $\mathcal{L}(\mathbf{u}, \mathbf{z})$ and $\mathcal{R}(\mathbf{u}, \mathbf{z})$ are defined by the square brackets on the LHS and RHS, respectively. The first three components of these vector valued operators are functions (of $\mathbf{x}$ ), whereas the rest that correspond to the lumped amplifier model are scalars.

\section{LINEARIZATION OF COUPLED PDES AND DAEs}

We now consider the linearization of the nonlinear operators in (1) around a given solution denoted by $\mathbf{u}_{p}(t, \mathbf{x})$ and by $\mathbf{z}_{p}(t)$. These linearizations are used for the numerical techniques based on Newton iterations, and in formulating the variational system that describes the perturbative and noise dynamics of (1) around a given time-varying solution. We compute the Jacobians of the nonlinear integro-differential operators $\mathcal{L}(\mathbf{u}, \mathbf{z})$ and $\mathcal{R}(t, \mathbf{u}, \mathbf{z})$ with respect to $\mathbf{u}$ and $\mathbf{z}$. We consider the Jacobian of $\mathcal{L}$ (same for $\mathcal{R}$ )

$$
\mathcal{J}_{\mathcal{L}}\left(\mathbf{u}_{p}, \mathbf{z}_{p}\right)=\left.\left[\begin{array}{c:c}
\frac{\partial}{\partial \mathbf{u}} \mathcal{L}_{\mathbf{u}}(\mathbf{u}, \mathbf{z}) & \frac{\partial}{\partial \mathbf{z}} \mathcal{L}_{\mathbf{u}}(\mathbf{u}, \mathbf{z}) \\
\hdashline \frac{\partial}{\partial \mathbf{u}} \mathcal{L}_{\mathbf{z}}(\mathbf{u}, \mathbf{z}) & \frac{\partial}{\partial \mathbf{z}} \mathcal{L}_{\mathbf{z}}(\mathbf{u}, \mathbf{z})
\end{array}\right]\right|_{\begin{array}{l}
\mathbf{u}=\mathbf{u}_{p} \\
\mathbf{z}=\mathbf{z}_{p}
\end{array}} .
$$

The Jacobian matrix above is not a simple matrix of scalar valued partial derivatives, some of its entries are in fact Fréchet derivatives [14], i.e., derivatives of operators with respect to functions. Its block entries have the following properties.

$$
\begin{aligned}
& \frac{\partial}{\partial t}\left[\begin{array}{c}
u_{1}\left(t, x_{1}\right) \\
\frac{E I}{Q_{K V} \omega_{Q}} \frac{\partial^{4}}{\partial x_{1}^{4}} u_{1}\left(t, x_{1}\right)+m u_{2}\left(t, x_{1}\right) \\
t_{d} u_{3}\left(t, x_{2}\right) \\
\mathbf{q}(\mathbf{z}(t))
\end{array}\right]=\left[\begin{array}{c}
u_{2}\left(t, x_{1}\right) \\
\left(-E I \frac{\partial^{4}}{\partial x_{1}^{4}}+T_{e} \frac{\partial^{2}}{\partial x_{1}^{2}}\right) u_{1}\left(t, x_{1}\right)-\frac{m \omega_{Q}}{Q_{V I}} u_{2}\left(t, x_{1}\right)+\mathbf{l}^{T} \mathbf{z}(t) \\
-\frac{\partial}{\partial x_{2}} u_{3}\left(t, x_{2}\right)
\end{array}\right] \\
& u_{1}\left(t, x_{1}\right)=0 \text { and } \frac{\partial}{\partial x} u\left(t, x_{1}\right)=0 \text { at } x_{1}=0, L \quad-\mathbf{f}\left(\mathbf{z}(t), u_{3}\left(t, x_{2}=1\right)\right)
\end{aligned}
$$


1) $\mathcal{J}_{\mathcal{L}_{\mathbf{u u}}}=(\partial / \partial \mathbf{u}) \mathcal{L}_{\mathbf{u}}: n \times n$ matrix valued linear operator. Operates on an $n \times 1$ vector of functions and produces an $n \times 1$ vector of functions.

2) $\mathcal{J}_{\mathcal{L}_{\mathbf{u z}}}=(\partial / \partial \mathbf{z}) \mathcal{L}_{\mathbf{u}}: n \times m$ matrix of functions. When multiplied with an $m \times 1$ vector of scalars, produces an $n \times 1$ vector of functions.

3) $\mathcal{J}_{\mathcal{L}_{\mathbf{z u}}}=(\partial / \partial \mathbf{u}) \mathcal{L}_{\mathbf{z}}: m \times n$ matrix valued linear functional. Maps an $n \times 1$ vector of functions to an $m \times 1$ vector of scalars.

4) $\mathcal{J}_{\mathcal{L}_{\mathbf{z z}}}=(\partial / \partial \mathbf{z}) \mathcal{L}_{\mathbf{z}}: m \times m$ matrix of scalars. Maps an $m \times 1$ vector of scalars to an $m \times 1$ vector of scalars.

In the linearization of (1), in addition to computing the Jacobians of the operators $\mathcal{L}$ and $\mathcal{R}$, we also need to linearize the $\mathrm{BCs}$ if they involve nonlinear operations.

\section{A. Linearization of the NEMS Oscillator Model}

We now consider the linearization of our case study, the NEMS oscillator captured by the coupled PDE-DAE system in (15), and the BCs in (16). The Jacobian of the operator $\mathcal{L}(\mathbf{u}, \mathbf{z})$ in $(15)$ is

$$
\mathcal{J}_{\mathcal{L}}(t)=\left[\begin{array}{ccc:c}
\mathcal{I} & \mathcal{O} & \mathcal{O} & 0 \\
\frac{E I}{Q_{K V} \omega_{Q}} \frac{\partial^{4}}{\partial x_{1}^{4}} & m \mathcal{I} & \mathcal{O} & 0 \\
\mathcal{O} & \mathcal{O} & t_{d} \mathcal{I} & 0 \\
\hdashline \mathscr{O} & \mathscr{O} & \mathscr{O} & \mathbf{C}(t)
\end{array}\right]
$$

where $\mathbf{C}(t)=\left.\frac{\partial}{\partial \mathbf{z}} \mathbf{q}(\mathbf{z})\right|_{\mathbf{z}=\mathbf{z}_{p}(t)}$

$\mathcal{I}: \quad$ identity operator: function $\rightarrow$ same function;

$\mathcal{O}: \quad$ zero operator: function $\rightarrow$ zero function;

$\mathscr{O}: \quad m \times 1$ vector of zero functionals: function $\rightarrow$ scalar zero;

0 : $\quad 1 \times m$ vector of zero functions;

$\mathbf{C}(t): m \times m$ matrix of scalars (for a given $t$ ).

Before we compute the Jacobian of $\mathcal{R}(\mathbf{u}, \mathbf{z})$ in (15), we define

$$
\begin{aligned}
\mathbf{G}_{\mathbf{z}}(t) & =\left.\frac{\partial}{\partial \mathbf{z}} \mathbf{f}\left(\mathbf{z}, u_{3}\right)\right|_{\mathbf{z}=\mathbf{z}_{p}(t), u_{3}=u_{p 3}\left(t, x_{2}=1\right)} \\
\mathbf{g}_{u_{3}}(t) & =\left.\frac{\partial}{\partial u_{3}} \mathbf{f}\left(\mathbf{z}, u_{3}\right)\right|_{\mathbf{z}=\mathbf{z}_{p}(t), u_{3}=u_{p 3}\left(t, x_{2}=1\right)} \\
\mathcal{D}_{T_{e}} & =\mathcal{T}_{e}\left(u_{p 1}\left(t, x_{1}\right)\right) \frac{\partial^{2}}{\partial x_{1}^{2}}+\left[\frac{\partial^{2}}{\partial x_{1}^{2}} u_{p 1}\left(t, x_{1}\right)\right] \mathcal{J}_{\mathcal{T}_{e}} \\
\mathcal{J}_{\mathcal{T}_{e}} & =\left.\frac{\partial}{\partial u_{1}} \mathcal{T}_{e}\left(u_{1}\right)\right|_{u_{1}=u_{p 1}\left(t, x_{1}\right)}
\end{aligned}
$$

where

$$
\begin{array}{ll}
\mathbf{G}_{\mathbf{z}}(t): & m \times m \text { matrix of scalars (for a } \\
& \text { given } t) ; \\
\mathbf{g}_{u_{3}}(t): & m \times 1 \text { vector of scalars (for a } \\
& \text { given } t \text { ); }
\end{array}
$$

$$
\mathscr{E}_{u_{3}\left(t, x_{2}=1\right)}
$$

$\mathcal{D}_{T_{e}}:$

$\mathcal{T}_{e}\left(u_{p 1}(t, x)\right):$

$\mathcal{J}_{\mathcal{T}_{e}}:$

$$
\left(\partial^{2} / \partial x_{1}^{2}\right) u_{p 1}\left(t, x_{1}\right)
$$

$\left[\left(\partial^{2} / \partial x_{1}^{2}\right) u_{p 1}\left(t, x_{1}\right)\right] \mathcal{J}_{\mathcal{T}_{e}}$

evaluation functional that acts on a function $\left[u_{3}\left(t, x_{2}\right)\right.$, for fixed $t$ as a function of $x_{2}$ ] and produces a scalar that is set to the value of the function at the specified evaluation point $\left(x_{2}=1\right)$;

linear operator with components as below, evaluated based on the chain/multiplication rules for differentiation;

scalar produced by the functional in (7);

linear functional $=$ Derivative of the nonlinear functional $\mathcal{T}_{e}$ : function $\rightarrow$ scalar;

for a given $t$, this is a function of $x_{1}$;

outer product of a function with a functional (for a given t). Functional $\mathcal{J}_{\mathcal{T}_{e}}$ : function $\rightarrow$ scalar. Overall an operator: function $\rightarrow$ function.

The Jacobian of $\mathcal{R}(\mathbf{u}, \mathbf{z})$ in (15) can then be computed as shown in (23), as shown at the bottom of this page. The Jacobians of $\mathcal{L}(\mathbf{u}, \mathbf{z})$ and $\mathcal{R}(\mathbf{u}, \mathbf{z})$ were computed using composition rules for operators, functionals, functions and scalars that are dictated by the chain and multiplication rules for Fréchet differentiation. The Fréchet derivative of the functional in (22) can be evaluated by several consecutive applications of the chain rule. The BCs in (16) are already linear, hence need not be linearized.

\section{Floquet Theory for Coupled PDEs And DAEs}

The variational system that is associated with (1) can be formulated based on the linearizations of the nonlinear operators and the BCs as follows:

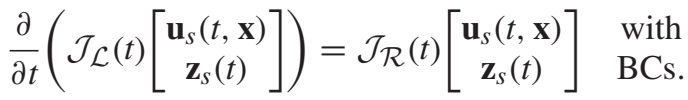

$\mathbf{u}_{s}$ and $\mathbf{z}_{s}$ represent the deviational (around the expansion point $\mathbf{u}_{p}$ and $\mathbf{z}_{p}$ ) distributed and lumped variables

$$
\mathbf{u}(t, \mathbf{x})=\mathbf{u}_{p}(t, \mathbf{x})+\mathbf{u}_{s}(t, \mathbf{x}), \quad \mathbf{z}(t)=\mathbf{z}_{p}(t)+\mathbf{z}_{s}(t) .
$$

The equations in (24) are a coupled set of linear PDEs and DAEs that represent a linear time-varying system. In the case when $\mathbf{u}_{p}(t, \mathbf{x})$ and $\mathbf{z}_{p}(t)$ are time-periodic, the variational system becomes linear periodically time-varying (LPTV). For the rest of our treatment in this section, we assume that $\mathbf{u}_{p}(t, \mathbf{x})$ and $\mathbf{z}_{p}(t)$ represent a time-periodic solution of (1) for the autonomous case when the RHS operator $\mathcal{R}(\mathbf{u}, \mathbf{z})$ does not have an explicit dependence on time $t$. The existence and the computation of a time-periodic autonomous solution for (1) is

$$
\mathcal{J}_{\mathcal{R}}(t)=\left[\begin{array}{ccc:c}
\mathcal{O} & \mathcal{I} & \mathcal{O} & 0 \\
-E I \frac{\partial^{4}}{\partial x_{1}^{4}}+\mathcal{D}_{T_{e}} & -\frac{m \omega_{Q}}{Q_{V I}} \mathcal{I} & \mathcal{O} & I \mathbf{l}^{T} \\
\mathcal{O} & \mathcal{O} & -\frac{\partial}{\partial x_{2}} & 0 \\
\hdashline \mathscr{O} & \mathscr{O} & -\mathbf{g}_{u_{3}}(t) \mathscr{E}_{u_{3}\left(t, x_{2}=1\right)} & -\mathbf{G}_{\mathbf{z}}(t)
\end{array}\right]
$$


a prerequisite for the Floquet analysis that is discussed below, as well as the derivation of the phase equation that will be covered in Section VI. In general, not all autonomous coupled DAE-PDE systems as in (1) will possess nontrivial timeperiodic solutions, i.e., autonomously oscillate. The ones that do will oscillate only for some settings of the system parameters. For instance, the feedback NEMS system autonomously oscillates only for a certain range of the feedback delay $t_{d}$ discussed in Section III-B.

Floquet theory is on a simple but extremely useful result on the structure of the solutions for LPTV systems [15]. It has ample applications in the analysis of the dynamics of oscillating systems. Floquet theory for systems that contain only lumped components, hence modeled either with a set of ordinary differential equations (ODEs) or DAEs, is well understood, see [16]. There exist generalizations of Floquet theory to PDEs [17].

We next describe a generalized form of Floquet theory that applies to hybrid (with both lumped and distributed components) LPTV systems in the form in (24). Instead of providing a formal proof for the results to be stated below, we outline here a straightforward, heuristic derivation technique that was advocated in [13] in another context: 1) we first discretize the space $\mathbf{x}$ dependence of the operators and distributed variables in (24) using an appropriate scheme; 2) this discretization turns (24) into a set of DAEs; 3) we can then apply the results of Floquet theory for DAEs [16] to the space discretized version of (24); and 4) finally, we take a limit to return to the continuous dependence on $\mathbf{x}$. Essentially, Floquet theory relates to the time dependence of LPTV systems. The nature of space dependence, whether it is discretized or continuous, is immaterial, apart from implications on the mechanics of setting up the theory. For instance, the definition of the dot product operation for vectors that contain both distributed and lumped variables is different than the case with only lumped variables, as we further discuss later below.

In extending Floquet theory from a DAE to a mixed DAE-PDE system, the discretization of the PDE component serves two purposes, one theoretical and the other one numerical/practical. In the theoretical case discussed above, discretization is used to extend the Floquet theory results from a DAE to a mixed DAE-PDE system, by first mapping from a distributed to a lumped system via discretization, then applying Floquet theory for DAEs, and finally going back by taking a limit to the continuum. In this theoretical use of discretization, one can use any sound convergent discretization scheme, e.g., finite-difference, finite-element, or spectral. However, a manually formed ad-hoc lumped model for the distributed components would be of no use in this case, since there is no well-defined procedure (which can be taken to the continuum limit) that allows one to go back and forth between the distributed and lumped domains. Once Floquet theory is extended as such, we arrive at a generalized form of Floquet theory expressed in functional form for mixed PDE-DAE systems. Then, for practical/numerical computations, one is again faced with a decision on how to discretize the PDE components, which is independent of what was used in extending the theory. In this case, one can use any form of discretization, including lumped models developed in an ad-hoc form. Finite-element and finite-difference discretizations are more flexible, they can handle complex geometries more easily, but they generate large sparse discretization matrices and have limited order of accuracy. On the other hand, spectral discretization offers superior accuracy for smooth problems. Ad-hoc lumped models can be very compact and computationally inexpensive, but they may miss important effects in the distributed component and there is no well defined procedure to obtain them.

The adjoint of the LPTV system in (24) is defined by

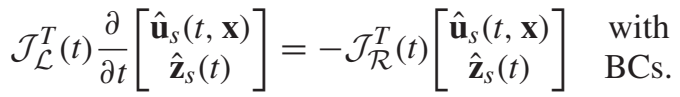

The modal solutions of (24) and (25) can be represented in the following form:

$$
\begin{aligned}
& \mathbf{u}_{s}(t, \mathbf{x})=\mathbf{u}_{s p}(t, \mathbf{x}) e^{\mu t} \quad \mathbf{z}_{s}(t)=\mathbf{z}_{s p}(t) e^{\mu t} \\
& \hat{\mathbf{u}}_{s}(t, \mathbf{x})=\hat{\mathbf{u}}_{s p}(t, \mathbf{x}) e^{-\mu t} \quad \hat{\mathbf{z}}_{s}(t)=\hat{\mathbf{z}}_{s p}(t) e^{-\mu t}
\end{aligned}
$$

where $\mu$ (a complex scalar) is called a Floquet exponent. $\mathbf{z}_{s p}(t)$ and $\hat{\mathbf{z}}_{s p}(t)$ are vectors of lumped variables that are periodically time-varying (PTV). The vector valued $\mathbf{u}_{s p}(t, \mathbf{x})$ and $\hat{\mathbf{u}}_{s p}(t, \mathbf{x})$ are also periodic in $t$, but also functions of $\mathbf{x}$. There are infinitely many such linearly independent modal solutions for (24) and (25), since the system is infinite-dimensional due to the distributed components. However, in practice, we can compute finitely many modal solutions once the distributed components are discretized. The Floquet exponents determine the stability properties of the variational system in (24) and its adjoint in (25). If all Floquet exponents have negative real parts, then one can conclude that $(24) /(25)$ is stable/unstable. If the variational system arises from a stable autonomous oscillator system, then one of the Floquet exponents satisfies $\mu=0$ due to the marginally stable nature of autonomous oscillators. We number the zero Floquet exponent as the first and denote the corresponding PTV quantities as $\mathbf{z}_{s p 1}(t), \mathbf{u}_{s p 1}(t, \mathbf{x}), \hat{\mathbf{z}}_{s p 1}(t)$, and $\hat{\mathbf{u}}_{s p 1}(t, \mathbf{x})$. One can easily show that [16]

$$
\mathbf{u}_{s p 1}(t, \mathbf{x})=\frac{\partial}{\partial t} \mathbf{u}_{p}(t, \mathbf{x}), \quad \mathbf{z}_{s p 1}(t)=\frac{d}{d t} \mathbf{z}_{p}(t)
$$

which means that the variational system in (24) has a PTV solution, which is (up to a scaling factor due to linearity) equal to the time derivative of the PTV solution of the original nonlinear system in (1). This holds only in the case when the system is autonomous, that is when the RHS operator $\mathcal{R}(\mathbf{u}, \mathbf{z})$ does not have an explicit dependence on $t$. The corresponding (for also $\mu=0$ ) PTV solution of the adjoint equation in (25), represented by $\hat{\mathbf{z}}_{s p 1}(t)$ and $\hat{\mathbf{u}}_{s p 1}(t, \mathbf{x})$, plays an important role in analyzing the behavior of autonomous oscillators [16]. It can be used to project the noise sources in order to quantify phase fluctuations and timing jitter. The solutions of (25) can be determined up to a scaling factor, due to linearity. $\hat{\mathbf{z}}_{s p 1}(t)$ and $\hat{\mathbf{u}}_{s p 1}(t, \mathbf{x})$ need to be properly normalized (scaled) in order for them to produce correct projections for the characterization of phase fluctuations

$$
\left[\begin{array}{c}
\hat{\mathbf{u}}_{s p 1}(t, \mathbf{x}) \\
\hat{\mathbf{z}}_{s p 1}(t)
\end{array}\right] \bullet\left(\mathcal{J}_{\mathcal{L}}(t)\left[\begin{array}{c}
\mathbf{u}_{s p 1}(t, \mathbf{x}) \\
\mathbf{z}_{s p 1}(t)
\end{array}\right]\right)=1
$$

The dot product operation above, denoted by $\bullet$, for a vector of both distributed and lumped variables is defined by

$$
\left[\begin{array}{c}
\mathbf{u}(t, \mathbf{x}) \\
\mathbf{z}(t)
\end{array}\right] \bullet\left[\begin{array}{c}
\mathbf{v}(t, \mathbf{x}) \\
\mathbf{y}(t)
\end{array}\right]=\mathbf{z}^{T}(t) \mathbf{y}(t)+\sum_{i=1}^{n} \int_{\mathbf{x} \in \mathbb{X}} u_{i}(t, \mathbf{x}) v_{i}(t, \mathbf{x}) d \mathbf{x}
$$


where $\mathbb{X}$ represents the spatial domain. The normalization condition in (29) needs to be enforced at some $t$ within one period. It will also hold for other values of $t[16]$.

\section{Vi. Phase Equation for Multidomain OSCILLATORS}

The role of the PTV solutions of the adjoint variational equation in (25) in characterizing phase fluctuations for autonomous oscillators is captured in a phase equation [18]. The essential and important dynamics of noisy oscillators can be encapsulated in a scalar phase variable $\alpha(t)$ [18]. For a multidomain autonomous oscillator, we have

$$
\frac{\partial}{\partial t} \mathcal{L}(\mathbf{u}, \mathbf{z})=\mathcal{R}(\mathbf{u}, \mathbf{z})+\mathbf{b}(t, \mathbf{x}) \quad \text { with BCs }
$$

where the disturbances $\mathbf{b}(t, \mathbf{x})=\left[\mathbf{b}_{\mathbf{u}}(t, \mathbf{x}), \mathbf{b}_{\mathbf{z}}(t)\right]^{T}$ are partitioned into ones that affect the distributed and lumped components. We can approximate the solution of (31) with

$$
\mathbf{u}(t, \mathbf{x}) \approx \mathbf{u}_{p}(t+\alpha(t), \mathbf{x}), \quad \mathbf{z}(t) \approx \mathbf{z}_{p}(t+\alpha(t))
$$

where the phase $\alpha(t)$ satisfies the phase equation given by

$$
\frac{d}{d t} \alpha=\left[\begin{array}{c}
\hat{\mathbf{u}}_{s p 1}(t+\alpha, \mathbf{x}) \\
\hat{\mathbf{z}}_{s p 1}(t+\alpha)
\end{array}\right] \bullet\left[\begin{array}{c}
\mathbf{b}_{\mathbf{u}}(t, \mathbf{x}) \\
\mathbf{b}_{\mathbf{z}}(t)
\end{array}\right] .
$$

$\mathbf{u}_{p}(t, \mathbf{x})$ and $\mathbf{z}_{p}(t)$ are the time-periodic solutions of (31) when the disturbance is removed. As apparent from the phase equation, the components of $\hat{\mathbf{z}}_{s p 1}(t)$ and $\hat{\mathbf{u}}_{s p 1}(t, \mathbf{x})$ quantify exactly how noise injected into the system at any time point within a period of oscillation, and at any spatial point of a distributed or lumped component, turn into phase fluctuations.

The phase equation in (33) can be derived using a generalization of a technique that was proposed in [13], where Novičenko and Pyragas considered oscillatory systems represented by a set of delay differential equations (DDEs), essentially ideal delay elements in addition to a set of ODEs. Since an ideal delay line is a distributed component, DDEs can be considered to be a very special case of the coupled PDE-DAE systems that we consider. Nevertheless, the "heuristic" method used by Novičenko and Pyragas [13] in deriving a phase model based on DDEs can be easily applied to the much more general case we consider here. In fact, we have already advocated the use of this technique in Section V in the generalization of Floquet theory from pure DAE to coupled DAE-PDE systems: 1) discretize the space dependence of the PDE-based components and combine them with the DAE-based ones to obtain a (larger) DAE system; 2) use the phase model derivation technique for pure DAE systems [16] on the discretized system; and 3) return to a coupled distributed-lumped representation by taking the limit so that the granularity of discretization becomes infinitesimal.

\section{NUMERICAL METHOdS AND Simulation TOOL}

The formulations above dictate difficult analysis problems and imply challenging issues that need to be addressed.

1) Syntactic description scheme for multidomain/physics systems with distributed and lumped components.

2) Automatic differentiation of a mixture of coupled nonlinear operators, functionals, and functions with respect to a set of distributed (function) and lumped (scalar) variables.

3) PSS analysis of driven and autonomously oscillating multidomain systems that are governed by a coupled set of PDEs and DAEs.

4) Floquet analysis of the variational system and its adjoint around a periodic solution computed by PSS analysis.

5) Stationary, cyclo-stationary, and phase noise analysis.

6) Efficient parameter sweeps for steady-state/noise analyses.

We developed new schemes, where appropriate, that address the above and implemented all of the numerical analysis modes in a MATLAB ${ }^{3}$ [19]-based prototype simulator for multidomain systems. Our current implementation supports 1-D spatial domains, but allows independent spatial coordinates for different physical domains. We were extremely fortunate to become aware of an open-source software package called Chebfun [20]. As stated in [20], "Chebfun extends MATLAB's inherent facilities with vectors and matrices to functions and operators." Chebfun provided us with most of the infrastructure needed for our simulator: 1) equation and $\mathrm{BC}$ specification format/parser; 2) automatic differentiation of nonlinear operators [14]; 3) representation of functions with Chebyshev interpolants on finite domains; and 4) automatic spectral collocation of spatial integro-differential linear operators and $\mathrm{BCs}$ that are compatible with Chebyshev representations [21]. However, the schemes and techniques available in Chebfun (version 5.3.0) were not able to address all of the analysis modes and issues identified above. We developed new formulations as detailed below, and implemented the following extensions as well as a number of substantial add-ons for our prototype simulator.

1) Extensions that enable handling a mixed set of distributed and lumped variables in system specification.

2) Extensions to the automatic differentiation infrastructure [14] in order to handle more general operations between operators, functionals, and functions that can also deal with mixed scalar/distributed variables. The manual derivative computations we have performed in Section IV can now be carried out in a completely automatic manner.

3) Numerical solution of initial value problems in time $t$ (i.e., time-stepping) for systems as in (1) via implicit time discretization schemes (e.g., multistep methods) that use analytically computed Jacobians as opposed to ones that are approximated with finite differences.

4) Representation of time-periodic distributed variables (functions of both $\mathbf{x}$ and $t$ ) with joint/hierarchical Chebyshev (Fourier) expansions for their spatial (time) dependence.

5) Numerical PSS analysis based on hierarchical Fourier spectral collocation in time and Chebyshev spectral collocation for space, which employs damped Newton iterations for solving the nonlinear equations, and preconditioned Krylov subspace-based iterative techniques for the solution of inner loop linear equations. For both forced and autonomous systems.

\footnotetext{
${ }^{3}$ MATLAB is a registered trademark of The MathWorks, Inc.
} 


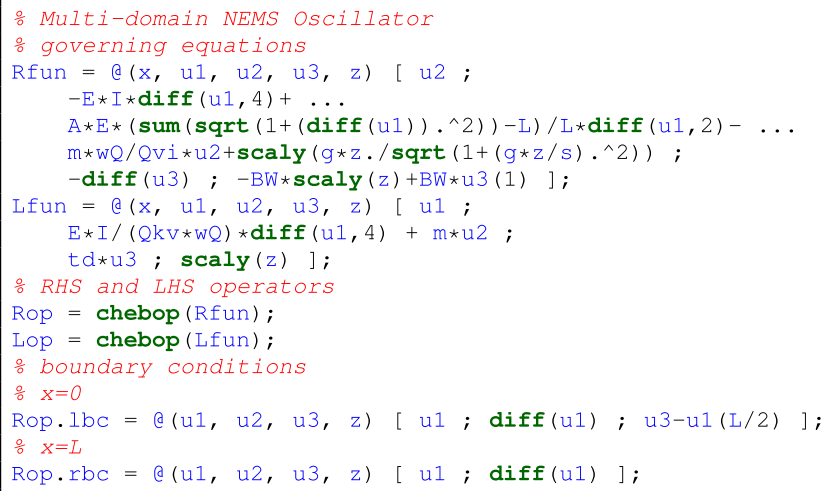

Listing 1. Multidomain NEMS oscillator.

6) Numerical, adaptive step-size, first-order pseudoarclength continuation [22] for performing drive frequency sweeps for PSS analysis of driven/forced multidomain systems.

7) Numerical Floquet analysis of the variational system around a periodic solution computed by PSS, which inherits the mixed hierarchical Fourier/Chebyshev spectral collocation scheme from PSS analysis mentioned above and employs Krylov subspace-based iterative techniques for solving the resulting eigenvalue problem.

8) Numerical, adaptive step-size, zero-order, simple (non arc-length) parameter continuation for performing parameter sweeps for PSS followed with Floquet analysis of autonomous multidomain oscillators.

9) Phase noise analysis for multidomain oscillators.

While some of the above can be considered as enhancements to the software infrastructure of Chebfun so that it can handle coupled DAE-PDE systems as opposed to pure PDE or ODE systems, there are others that necessitated the development of novel formulations and required nontrivial implementations. Next, we elaborate on the most important ones.

\section{A. System Description and Equation Specification}

The MATLAB code in Listing 1 captures the equations and the BCs for the NEMS oscillator in (15) and (16).

The amplifier model is chosen as a saturating amplifier [23]

$$
\begin{aligned}
& \frac{d}{d t} z=-B W z+B W b(t) \\
& y(t)=\frac{g z(t)}{\sqrt{1+\left(\frac{g z(t)}{s}\right)^{2}}}
\end{aligned}
$$

where $B W$ is the bandwidth, $b(t) / y(t)$ is the input/output, $g$ is the gain for low frequencies and small inputs, and $s$ is the output saturation level. Most of the code in Listing 1 is self explanatory. Chebop is a Chebfun utility for defining operators. Scaly is a syntactic utility we have implemented as an extension to Chebfun that specifies its argument to be scalar valued, i.e., not a function of $x$.

\section{B. Chebyshev and Fourier Representations of Variables}

The lumped variables $\mathbf{z}(t)$ in (1) depend on $t$ only, whereas the distributed variables $\mathbf{u}(t, \mathbf{x})$ depend both on $t$ and $\mathbf{x}$. In the transient analysis of multidomain systems based on timestepping schemes, the representation of the time dependence is straightforward, i.e., discrete time points selected by the multistep solver. In order to represent the spatial dependence of the distributed variables for a given $t$, we use Chebfun. A chebfun for a function on a finite domain is a polynomial interpolant through Chebyshev points [20], [24]. Chebfun provides efficient implementations for various operations, such as evaluation, differentiation, integration, etc. based on fast algorithms [20], [24].

In PSS, Floquet and phase noise analyses, the time dependence of the system variables is periodic. For the lumped variables, we use Fourier expansions. However, the representation of the distributed variables in the time-periodic case is not obvious. Chebfun was recently extended to functions of more than one variable [25]-[28]. However, it is not possible to mix Fourier and Chebyshev expansions for multivariable functions as we require, it is either all Chebyshev or all Fourier. Efficient construction of representations, and their numerical manipulation, for multivariate functions are open problems. We use a relatively simple approach currently, by combining a Chebyshev interpolant with a Fourier series as a tensor product [28]

$$
u(x, t)=\sum_{i=0}^{N}\left[\sum_{k=-K}^{K} c_{i k} e^{j k \omega_{p} t}\right] p_{i}(x)
$$

with the fundamental frequency $\omega_{p}=2 \pi / T_{p}$ and

$$
\sum_{k=-K}^{K} c_{i k} e^{j k \omega_{p} t}=u\left(x_{i}, t\right)
$$

where $x_{i}$ 's are the Chebyshev points, $x_{i}=\cos (i \pi / N)$ (assuming a domain $[-1,1]$ ), and $p_{i}(x)$ 's are the Lagrange basis functions for the polynomial interpolant through $x_{i}$ [24]. For a Chebyshev point $x_{i},(37)$ is a Fourier representation for $u\left(x_{i}, t\right)$. The coefficients $c_{i k}$ for $u\left(x_{i}, t\right)$ are computed (via an FFT) by sampling $u\left(x_{i}, t\right)$ in $t$ with uniformly spaced time points at $t_{l}=(l-1) /(2 K+1) T_{p}$ for $l=1, \ldots, 2 K+1$. One can go back and forth between uniformly spaced time samples and coefficients via FFTs and perform various operations efficiently using the appropriate (sample or coefficient-based) representation. For a given $t_{l}$ on the other hand, the interpolant in (36) is equivalent to the Chebyshev interpolant

$$
u\left(x, t_{l}\right)=\sum_{i=0}^{N} u\left(x_{i}, t_{l}\right) p_{i}(x)=\sum_{i=0}^{N} \hat{c}_{i}\left(t_{l}\right) \mathrm{T}_{i}(x)
$$

where $\mathrm{T}_{i}$ is the degree $i$ Chebyshev polynomial [24]. The coefficients $\hat{c}_{i}\left(t_{l}\right)$ are computed from the function values at the Chebyshev points, i.e., $u\left(x_{i}, t_{l}\right)$ 's. Similarly, the transformations between sample and coefficient-based representations are done via FFTs.

\section{Periodic Steady-State Analysis for Coupled DAE-PDEs}

For the PSS analysis of a coupled PDE-DAE system, we need to solve the following mixed-boundary value problem:

$$
\begin{aligned}
\frac{\partial}{\partial t} \mathcal{L}(\mathbf{u}, \mathbf{z}) & =\mathcal{R}(t, \mathbf{u}, \mathbf{z}) \quad \text { with } \\
\mathbf{u}(t, \mathbf{x}) & =\mathbf{u}\left(t+T_{p}, \mathbf{x}\right), \mathbf{z}(t)=\mathbf{z}\left(t+T_{p}\right), \text { BCs in } \mathbf{x}
\end{aligned}
$$

both in the forced and autonomous cases. 
We formulate the analysis based on the joint ChebyshevFourier representation in (36). We use the Chebyshev technology of Chebfun, but implemented our own Fourier technology as an add-on for a joint/hierarchical Chebyshev-Fourier technology. Chebysev spectral collocation is used for the $\mathbf{x}$, and Fourier spectral collocation for the $t$ dependence in discretizing the mixed-boundary problem in (39). When a joint Chebyshev-Fourier collocation-based discretization is applied to (39), a system of nonlinear algebraic equations is obtained. For a problem with a 1-D spatial domain, the size of this algebraic equation system is $(n N+m) L$, where $n(m)$ is the number of distributed (lumped) variables. $N$ is the number of the terms in the Chebyshev interpolants, and $L=2 K+1$ is the number of terms in the Fourier representation. We solve this set of nonlinear algebraic equations using damped Newton iterations. The initial guess for the Newton iterations is generated by running a transient analysis. In every iteration of Newton's method, a linear system of equations, which has the same size as the nonlinear equation set, needs to be solved. We solve this system using GMRES [29] as implemented in MATLAB. The large matrix with size $(n N+m) L$ is never formed, but an implicit routine that multiplies it with a vector is used. In this routine and in evaluating the RHS of the set of nonlinear equations, we use FFTs in order to go back and forth between the coefficient and sample domains. In order to accelerate the GMRES iterations, we use a block diagonal preconditioner [30]. The discretization of (39) with the joint Chebysev-Fourier scheme is performed in a hierarchical manner so that the discrete variables (i.e., Fourier coefficients of the values of the distributed variables at the Chebyshev points and the lumped variables) are sorted in a Fouriermajor, Chebyshev-minor manner. As such, the block diagonal preconditioner corresponds to ignoring the mixing between the Fourier components that arises due to the nonlinear effects in the system. In our current implementation, we fully exploit the implicit structure in the large Jacobian discretization matrix that arises due to Fourier collocation of time dependence. Currently, any structure that may be due to Chebyshev discretization of the space dependence is exploited only to the extent that it results in straightforward sparsity in matrix instantiations of the linear spatial operators via Chebyshev collocation. For instance, if a model contains distributed components in two different physical domains which are coupled to each other only through interface components (i.e., BCs), this results in sparsity (zero blocks) in the discretized matrix representations of the spatial operators. Lumped components are also typically interfaced with the distributed components through interface components represented by BCs. However, unlike finite difference schemes, most spectral discretization techniques generate dense blocks [4] for operators acting on distributed variables. As a result, the computational complexity of PSS analysis in our current implementation is roughly $O\left(\left(n N^{3}+m\right) L\right)$, assuming that the lumped part of the system has sparse interactions, and the distributed components and variables are coupled with each other through only BCs. On the other hand, there is no inherent theoretical obstacle to exploiting all of the implicit structure in a hierarchical, joint Chebyshev-Fourier collocation technique, rendering it fully matrix-free. The linear spatial operators do not need to be instantiated as full matrices, as exemplified in [31].
The fully matrix-free version of the hierarchical FourierChebyshev collocation scheme for PSS analysis would result in a computational complexity of $O((n N+m) L)$. Finally, in the ultimate matrix-free version of PSS analysis for DAEPDE systems, one may be able to use compressed low-rank representations [26] (as opposed to simple tensor products) for joint Chebyshev-Fourier representations and achieve an ultimate analysis complexity of $O((N+L) n+L m)$.

\section{Floquet Analysis for Coupled LPTV DAEs and PDEs}

The form of the modal solutions of the coupled LPTV PDEDAE system in (24) are as in (26) as dictated by Floquet theory. We substitute (26) into (24) to obtain

$$
\begin{aligned}
\mu \mathcal{J}_{\mathcal{L}}(t)\left[\begin{array}{c}
\mathbf{u}_{s p}(t, \mathbf{x}) \\
\mathbf{z}_{s p}(t)
\end{array}\right]= & \left(\mathcal{J}_{\mathcal{R}}(t)-\frac{\partial}{\partial t} \mathcal{J}_{\mathcal{L}}(t)\right)\left[\begin{array}{c}
\mathbf{u}_{s p}(t, \mathbf{x}) \\
\mathbf{z}_{s p}(t)
\end{array}\right] \\
& \text { with BCs. }
\end{aligned}
$$

The above defines a generalized eigenvalue problem, which can be solved to compute the Floquet modes of (24). The linear but time-periodic operators $\mathcal{J}_{\mathcal{L}}(t)$ and $\mathcal{J}_{\mathcal{R}}(t)$ are the linearizations of the nonlinear operators $\mathcal{L}(\mathbf{u}, \mathbf{z})$ and $\mathcal{R}(\mathbf{u}, \mathbf{z})$ around the time-periodic solution $\mathbf{u}_{p}(t, \mathbf{x}), \mathbf{z}_{p}(t)$ that can be numerically computed by PSS analysis. In order to solve the eigenvalue problem in (40), we inherit the same joint Fourier-Chebyshev discretization and the hierarchical spectral collocation scheme that are used for PSS analysis. In solving the discretized eigenvalue problem, we use the eigs utility in MATLAB, which is based on Arnoldi iterations. Just as GMRES is used in the PSS analysis in a matrix-free manner, we use eigs in a matrix-free manner in order to compute several Floquet eigenmodes that have the Floquet exponents with the smallest magnitude. In doing so, Arnoldi iterations are actually performed using a shift-invert scheme, where the invert operations are implicitly performed via preconditioned GMRES [32]. The computations outlined above can also be performed on the adjoint system defined by (25). The Floquet eigenmode of the adjoint system computed as such that corresponds to the zero Floquet exponent is then normalized using (29) and (30). This eigenmode serves as the key in characterizing phase noise for multidomain oscillators, which we discuss next.

\section{E. Phase Noise Analysis for Multidomain Oscillators}

Phase noise characterization for multidomain oscillators is performed based on the phase equation in (33) and the eigenmode of the adjoint system that corresponds to the zero Floquet exponent, represented by $\hat{\mathbf{z}}_{s p 1}(t)$ and $\hat{\mathbf{u}}_{s p 1}(t, \mathbf{x})$. The stochastic analysis and characterization of phase noise based on the phase equation proceeds along the lines of the analysis described in [16], [33], and [34] for purely lumped ODE and DAE systems. Instead of repeating essentially the same analysis here, we would like to emphasize what is different.

With a system that contains distributed components, the noise sources represented by $\mathbf{b}_{\mathbf{u}}(t, \mathbf{x})$ in (33) also have a distributed nature. As such, their characteristics need to be specified in terms of not only their temporal behavior but also spatially. For instance, a white noise source may be white in the temporal sense, i.e., with uncorrelated time samples, and/or in space, i.e., with uncorrelated values at two different spatial points of the distributed component. 
Unlike purely lumped systems, the equations for the coupled DAE-PDE systems have associated BCs for the spatially distributed components. Even though the BCs are implicit in (1), (24), and (25), they become instantiated as algebraic constraints when these equations are discretized based on Fourier and/or Chebyshev collocation. This also holds for the eigenvalue problem in (40), and for the similar problem for the adjoint system in (25). Thus, as we compute $\hat{\mathbf{z}}_{s p 1}(t)$ and $\hat{\mathbf{u}}_{s p 1}(t, \mathbf{x})$ for the adjoint system, we also obtain adjoint components that correspond to the BCs of the forward system in (24). At first thought, these components may seem like abstract necessities of the Fourier-Chebyshev collocation used in solving the eigenvalue problems. However, these components do have a deeper physical meaning. Just as the components of $\hat{\mathbf{z}}_{s p 1}(t)$ and $\hat{\mathbf{u}}_{s p 1}(t, \mathbf{x})$ quantify exactly how noise injected into the system turns into phase fluctuations, the components that correspond to the $\mathrm{BCs}$ for the adjoint system quantify how any disturbance to the $\mathrm{BC}$ s creates phase fluctuations. This can be best understood by noting that the adjoint system in (25) is obtained from the forward system in (24) by transposing the matrix valued operators $\mathcal{J}_{\mathcal{L}}(t)$ and $\mathcal{J}_{\mathcal{R}}(t)$ : there is a one-to-one correspondence between the variables of the adjoint system and the equations of the forward system. That is why the impact of noise (injected into an equation) on phase fluctuations is characterized by the corresponding variable of the adjoint system. BCs of the forward system appear as (algebraic) equations when the system is discretized and instantiated with matrices. There is in fact a corresponding adjoint system variable for every BC equation.

\section{RESULTS}

We present numerical analysis results on our case study. The ultimate limits to the sensitivity of mass sensors based on NEMS oscillators are determined by inherent fluctuations and noise in the mechanical and electrical domain [10], [35], [36]. Recent work on the analysis of NEMS oscillator mass sensors [23], [37], [38] based on lumped models revealed that when the mechanical resonators are operated at specific points in the nonlinear regime they offer better noise performance. We investigate this issue using our proposed hybrid distributed-lumped analysis approach and simulation tool.

\section{A. Setup and System Parameters}

We use the parameter values and the geometrical dimensions given in [10] for the mechanical beam resonator. However, for our numerical calculations, we use normalized (scaled) quantities. We normalize the length of the beam to be unity, its width and length are scaled accordingly. We use a mass scaling factor set to an attogram. Time is scaled in such a way so that the smallest modal vibration frequency for the linear, lossless beam is equal to normalized 1 . The scaling factors are summarized in Table I. The scaled beam parameters are given in Table II. Other system parameters, such as the quality factors for the resonator, the amplifier parameters and the delay line characteristics vary depending on the analysis scenario. They will be specified, where appropriate.
TABLE I

SCALING FACTORS

\begin{tabular}{|c|c|c|}
\hline $\begin{array}{c}\text { Length } \\
\mu \mathrm{m}\end{array}$ & $\begin{array}{c}\text { Time } \\
\text { nanosecs }\end{array}$ & $\begin{array}{c}\text { Mass } \\
\text { attogrs }\end{array}$ \\
\hline \hline 0.66 & 0.99516 & 1 \\
\hline
\end{tabular}

TABLE II

SCALEd (Normalized) Beam Parameters

\begin{tabular}{|c|c|c|}
\hline $\begin{array}{c}L \\
\text { (length) }\end{array}$ & $\begin{array}{c}t \\
\text { (thickness) }\end{array}$ & $\begin{array}{c}w \\
\text { (width) }\end{array}$ \\
\hline \hline 1 & 0.0758 & 0.0758 \\
\hline \hline $\begin{array}{c}m \\
\text { (mass/length) }\end{array}$ & $\begin{array}{c}E \\
\text { (Young's modulus) }\end{array}$ & $\begin{array}{c}I \\
\text { (Inertia) }\end{array}$ \\
\hline 3.8445 & $1.1046 \times 10^{5}$ & $2.7449 \times 10^{-6}$ \\
\hline
\end{tabular}

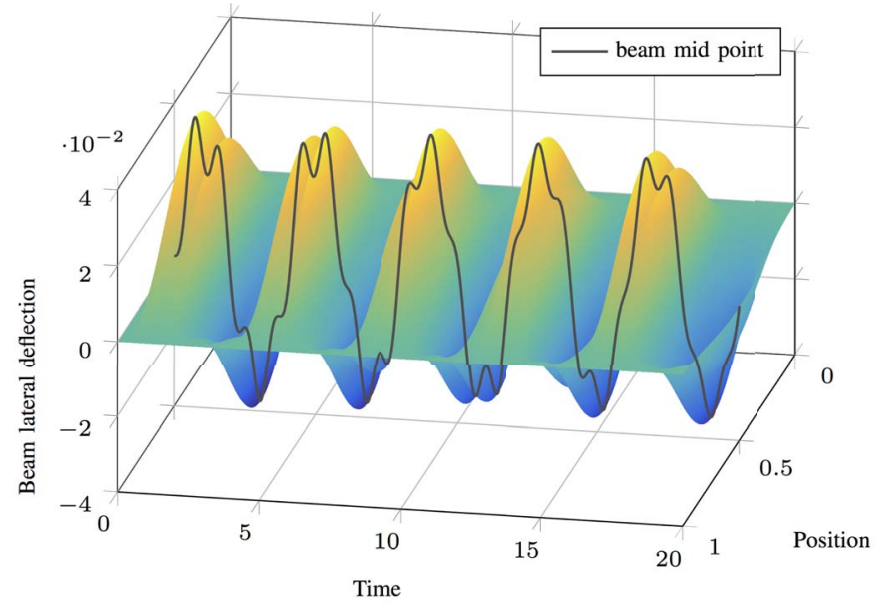

Fig. 2. Open loop beam resonator: transient analysis.

\section{B. Transient and PSS Analysis: Driven Beam Resonator}

The beam resonator in this case is operated in an open-loop configuration based on the equations in (13) and (14), with the $\mathrm{BCs}$ in (4). We include loss in the beam with quality factors $Q_{K V}=2000$ and $Q_{V I}=2000$, with an overall quality factor of $Q=1000$. We also take into account the nonlinear effect due to tension as captured by a term in (14). The excitation is chosen as below with $A=1$ and $f_{c}=0.25$ (normalized)

$$
a\left(t, x_{1}\right)=A \sin \left(2 \pi f_{c} t\right) .
$$

For transient analysis, the initial conditions are set to zero at all points along the beam, i.e., $u_{1}\left(t=0, x_{1}\right)=0$ and $u_{2}\left(t=0, x_{1}\right)=0$ for $0 \leq x_{1} \leq 1$. We use an implicit second-order time-stepping scheme, and 29-point Chebyshev representations for $u_{1}\left(t, x_{1}\right)$ and $u_{2}\left(t, x_{1}\right)$ at a given $t$. Fig. 2 shows the transient analysis result for the lateral deflection $u_{1}\left(t, x_{1}\right)$ of the beam as a function of both position $x_{1}$ and time $t$. On this 3-D plot, the deflection for the mid-point (at $x_{1}=L / 2$ ) of the beam is traced with a curve. With a sinusoidal excitation as in (41), the beam resonator eventually reaches a time-PSS, which can be directly computed with a PSS analysis. As described in Section VII-C, the PSS analysis is performed by a hiearchical Fourier-Chebyshev spectral technique. The initial guess for the Newton iterations is obtained via transient analysis. A Fourier-Chebyshev representation as in (36) with 29 Chebyshev points and 31 Fourier components was used for $u_{1}\left(t, x_{1}\right)$ and $u_{2}\left(t, x_{1}\right)$. The total number of 


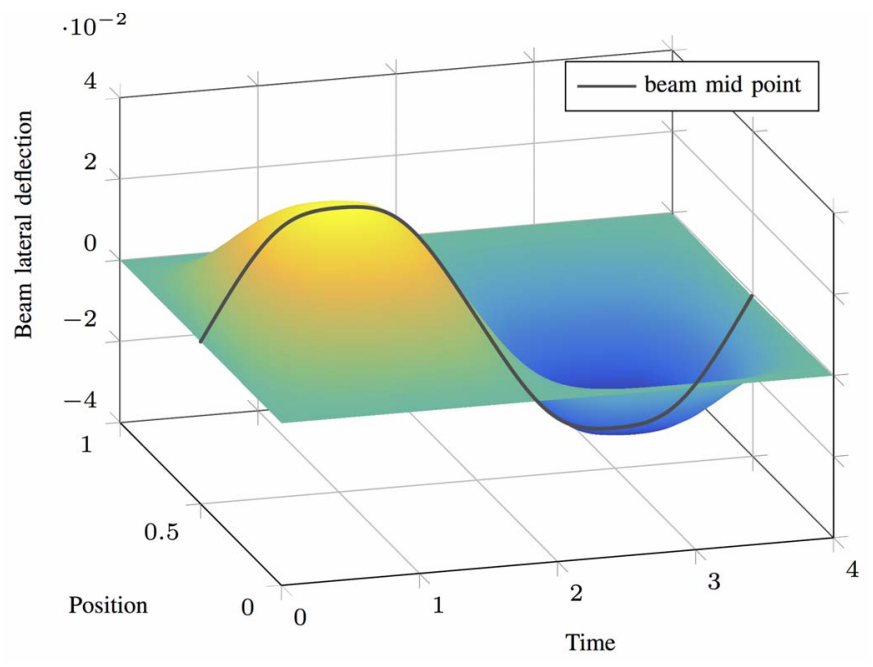

Fig. 3. Open loop beam resonator: PSS analysis.

nonlinear algebraic equations obtained via Fourier-Chebyshev spectral discretization is equal to $(2 \times 29+4) \times 31=1922$. Four additional equations are needed in order to capture the spatial BCs. With rectangular spectral collocation [21], we have effectively used a $29+4=33$ point Chebyshev interpolant for the $x_{1}$ dependence of $u_{1}\left(t, x_{1}\right)$, which is projected to 29 collocation points. With the initial guess from transient analysis, the Newton iterations converge quadratically to the desired relative accuracy $\left(10^{-8}\right)$ within four iterations. The time-PSS for the lateral deflection $u_{1}\left(t, x_{1}\right)$ is shown in Fig. 3. We observe that the beam steady-state is far from being sinusoidal in time $t$, due to the nonlinear effect in the beam.

\section{Frequency Sweep for PSS Analysis: Driven Beam Resonator}

The beam resonator is operated in an open-loop configuration, with a sinusoidal drive as in (41). We include the nonlinear effect due to tension, and also loss due to viscous damping with $Q_{V I}=10$ but set damping due to the Kelvin-Voigt effect to zero. The analysis is carried out for a range of drive amplitudes, from $A=0.01$ to $A=3.00$. We perform the drive frequency sweep for PSS analysis using the arclength continuation technique. Fig. 4 shows the RMS value (over one period in $t$ ) of the beam lateral deflection at its mid-point as a function of drive frequency for a range of drive amplitudes. Each point on the family of graphs in Fig. 4 represents a PSS analysis. However, the initial guesses for these analyses are not all generated via transient analyses, they are in fact generated from a previous run of the analysis via first-order continuation. As seen in Fig. 4, at larger drive amplitudes that excite the nonlinearity of the beam, there are multiple solutions for a range of drive frequencies. The arclength continuation scheme enables us to compute all of these multiple solutions. However, not all of these solutions are stable. The behavior revealed by the results in Fig. 4 is well-known and appears in the periodically forced nonlinear duffing oscillator [39]-[41]. In fact, the scalar duffing model is used as a simple representation for mechanical beam resonators [41]. Our results in Fig. 4 reveal a curious effect: at the highest drive amplitudes, apart from the main multivalued

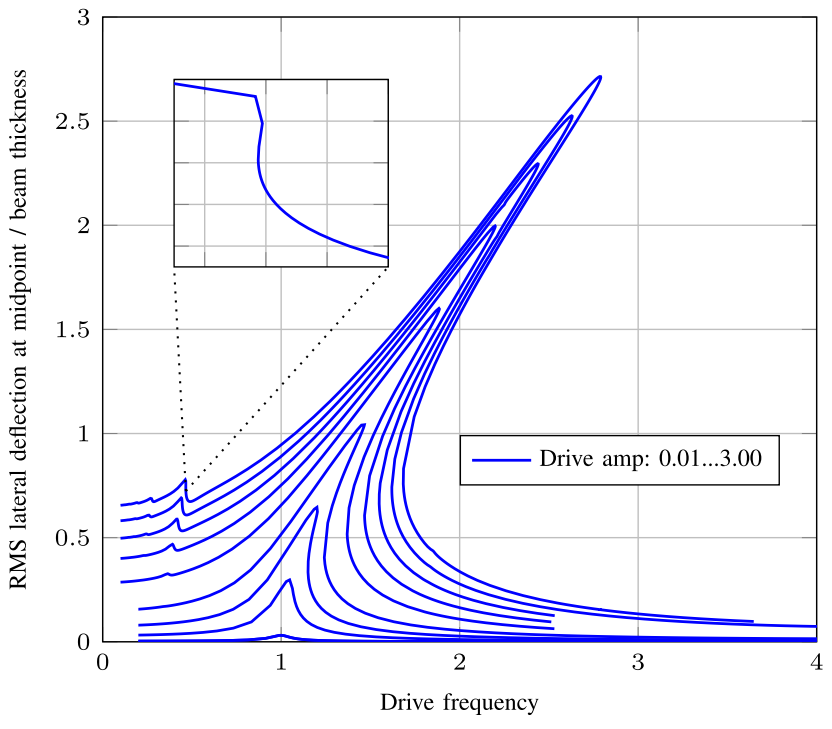

Fig. 4. Open loop beam resonator: frequency sweep PSS.

branch of the frequency response at frequencies larger than 1 , one can observe a smaller scale secondary multivalued (blown up in the figure for better visibility) branch at around a frequency value of 0.46 . In fact, there is a tertiary branch (which is not multivalued) that is visible as a tiny blip at around a frequency value of 0.27 . We believe that these secondary and tertiary peaks in the frequency response are a manifestation of the superharmonic resonance phenomenon [39] seen in forced nonlinear oscillatory systems.

1) Comparison With Previous Work: Results similar to the ones above are reported in [42] and [43]. These works are on the numerical solution of nonlinear equations for distributed beam models using a modal/finite-element technique [42], and a spectral Chebyshev one [43]. Especially the work in [43] is noteworthy: a numerical technique that is based on a Chebysev representation for spatial dependence is proposed. However, instead of a collocation scheme [21], a Galerkin scheme is used. Even though the handling of the BCs is achieved with a projection scheme as in [21], their approach seems less general. The rectangular spectral collocation approach [21] we use is straightforward. Nevertheless, it appears that in both works [42], [43], the time-PSS is computed by running a transient time-stepping scheme long enough, as opposed to the hierarchical Fourier-Chebyshev technique we have proposed that computes the steady-state solution directly and efficiently. Furthermore, they do not employ an arclength continuation scheme in tracing the frequency response curves with folds. They seem to be performing a simple drive frequency sweep, where at each frequency a transient analysis is run anew. This must be quite costly computationally, and cannot capture the unstable branches. That is probably why the frequency response curves presented in [42] and [43] exhibit jumps at the fold locations.

\section{Delay Sweep for PSS Analysis: NEMS Feedback Oscillator}

We include loss in the beam with quality factors $Q_{K V}=20$ and $Q_{V I}=20$, with an overall quality factor of $Q=10$. 


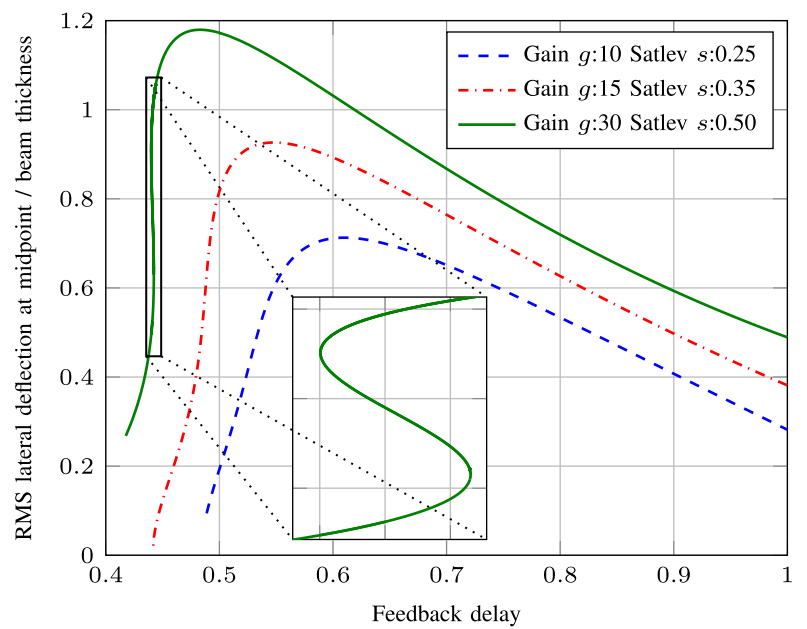

(a)

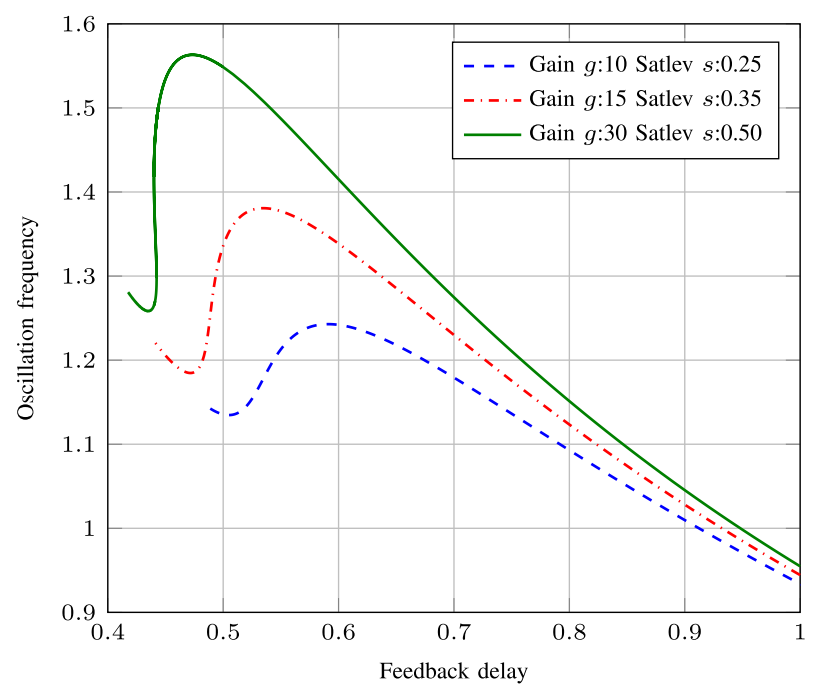

(b)

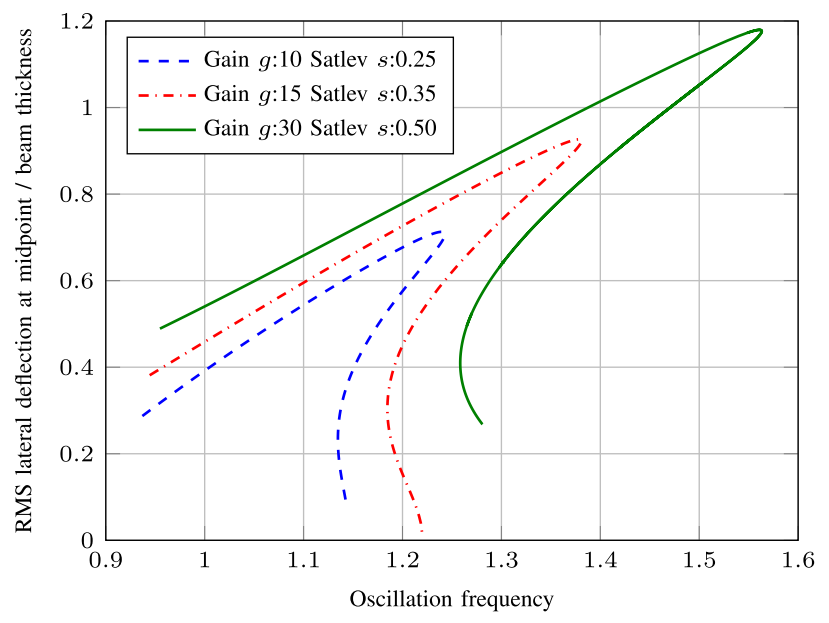

(c)

Fig. 5. NEMS feedback oscillator: delay sweep PSS. (a) RMS lateral deflection versus feedback delay. (b) Oscillation frequency versus feedback delay. (c) RMS lateral deflection versus oscillation frequency.

The model for the amplifier is as specified in Section VII-A: a simple saturating amplifier. We choose the bandwidth BW of the amplifier to be very large. This means that $z(t)=b(t)$ in (34). We consider three sets of values for the small amplitude gain $g$ and the output saturation level $s$ for the amplifier, as defined in (35). Fig. 5(a) shows the RMS beam lateral deflection at its mid-point, as a function of feedback delay, for the three amplifier parameter sets. Each point on the family of graphs in Fig. 5(a) represents the result of a PSS analysis for the autonomous NEMS oscillator at a certain feedback delay setting. We observe that, for each amplifier parameter set, the NEMS resonator oscillates with maximum amplitude at a certain value of feedback delay. More interestingly, for the largest gain setting of the amplifier, the NEMS oscillator exhibits multiple oscillatory solutions for a narrow range of feedback delay values. We are not aware of any other work in the literature that has demonstrated this behavior for an NEMS feedback oscillator, experimentally, or computationally. The oscillation frequency at each feedback delay setting is determined by the autonomous dynamics of the oscillator. In PSS analysis, the oscillation frequency is an unknown, and is solved for along with the other variables. Fig. 5(b) shows the oscillation frequency as a function of feedback delay. The data for this plot comes from the same set of PSS analyses that were run to generate the data for Fig. 5(a). We observe that the oscillation frequency as a function of feedback delay exhibits two local extrema, a maximum, and a minimum. These two points are of interest [23], [37], [38], for the phase noise performance of the oscillator, to be discussed in the next section. The feedback delay value that corresponds to the maximum frequency in Fig. 5(b) seems to be the same as the delay value that corresponds to the maximum amplitude in Fig. 5(a). We can use the same data in these plots in order to create a plot of the RMS value of the beam lateral deflection versus frequency, shown in Fig. 5(c). We observe that the maximum frequency indeed corresponds to the maximum amplitude. This plot looks similar to Fig. 4 for the open-loop, driven resonator. However, all of the solutions represented in Fig. 5(c) are stable, autonomous solutions of the feedback oscillator, whereas not all solutions in Fig. 4 correspond to stable ones for the driven, open-loop resonator. As discussed in [37], "unstable solutions in the open-loop configuration are stabilized by the closed-loop feedback."

\section{E. Delay Sweep for Phase Noise Analysis: NEMS Oscillator}

The sensitivity of NEMS oscillators used as ultrasensitive sensors is determined by their noise performance [10], [35]. There are various noise sources in NEMS systems. We concentrate on the two most important ones: 1) electronic amplifier noise and 2) mechanical resonator noise.

1) Impact of Amplifier Noise on Phase Noise: The major source of noise in the electronic domain is the amplifier. For the simple saturating amplifier in (34) and (35), we use a white noise source at the input to model its noise contribution. The impact of this input-referred noise source on the phase noise performance of the NEMS oscillator can be quantified via the time-periodic Floquet eigenmode $\hat{z}_{s p 1}(t)$ of the adjoint equation, as discussed in Section V. Based on the results in [34], the contribution and impact of amplifier noise on phase noise can be computed as follows $\mathrm{PN}_{\mathrm{amp}}=\int_{t=0}^{T_{p}}\left[\hat{z}_{s p 1}(t)\right]^{2} d t$. $\mathrm{PN}_{\mathrm{amp}}$ is normalized, in the sense that the strength of the 


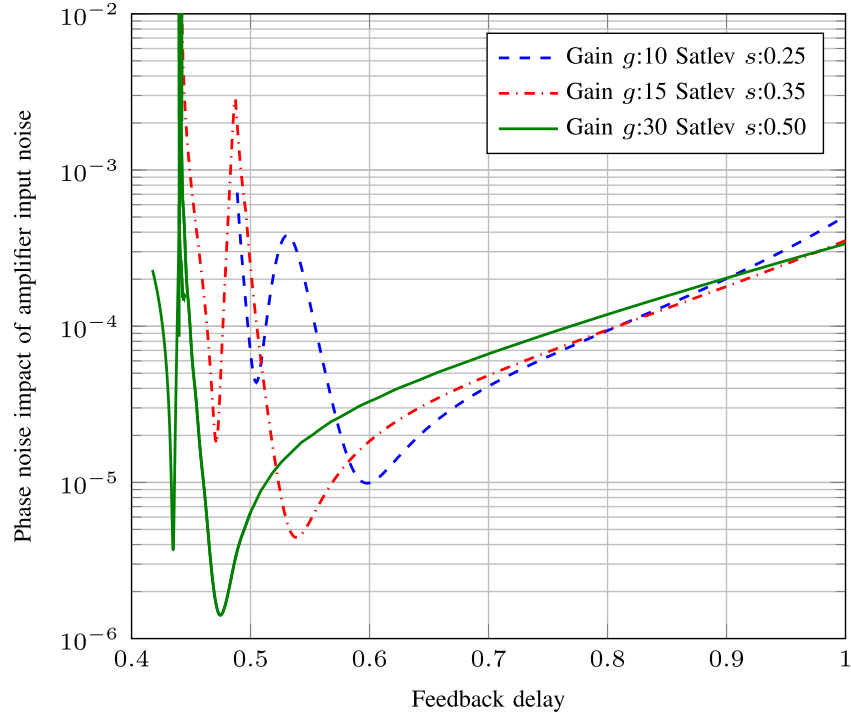

Fig. 6. Phase noise impact of amplifier noise.

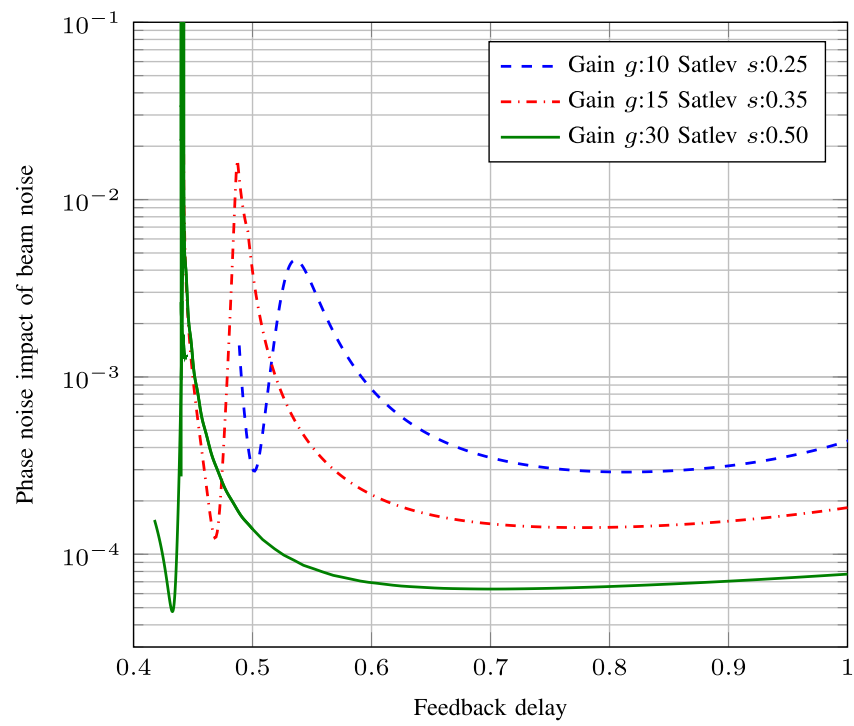

Fig. 7. Phase noise impact of distributed beam noise.

noise source is set to unity. Using the techniques discussed in Sections VII-D and VII-E, $\mathrm{PN}_{\mathrm{amp}}$ can be computed for a certain feedback delay setting based on PSS and Floquet analyses. Fig. 6 shows $\mathrm{PN}_{\mathrm{amp}}$ as a function of feedback delay, for the three amplifier parameter sets. We observe that the phase noise impact of amplifier noise has two sharp local minima. These minima in fact correspond to the two turning points in Fig. 5(c). The minimum on the right in Fig. 6 corresponds to the first turning point in Fig. 5(c) with maximum frequency and amplitude, whereas the one on the left corresponds to the second turning point with smaller frequency and amplitude. Both of the minima become deeper as the saturation level of the amplifier increases.

2) Impact of Mechanical Beam Noise on Phase Noise: The fluctuation-dissipation theorem dictates that every dissipation mechanism is associated with a noise source [10]: every loss mechanism in the beam resonator corresponds to a noise source. The mechanical noise due to intrinsic losses in the beam material and viscous damping is white, similar to thermal noise in electrical circuits, however, with a distributed nature as the beam itself. As discussed in [10], this distributed noise source has uncorrelated samples not only in time but also in space: the mechanical noise at two different points on the beam are uncorrelated. The impact of this distributed noise source on the phase noise performance of the NEMS oscillator can be quantified via the time-periodic Floquet eigenmode $\hat{u}_{s p 1}(t, x)$ as follows. $\mathrm{PN}_{\text {beam }}=\int_{t=0}^{T_{p}} \int_{x=0}^{1}\left[\hat{u}_{s p 1}(t, x)\right]^{2} d x d t$. The integral with respect to $x$ is due to the distributed nature of beam noise. Fig. 7 shows $\mathrm{PN}_{\text {beam }}$ as a function of feedback delay, for the three amplifier parameter sets. We observe that the phase noise impact of mechanical beam noise also has two local minima. However, the local minimum on the right in Fig. 7 is not sharp, and does not correspond to the first turning point in Fig. 5(c).

3) Comparison With Previous Work: The noise analysis results reported above for an NEMS resonator-based feedback oscillator qualitatively agree with previous results reported in the literature. The "noise quenching effect" [23] for the amplifier noise, with a saturating amplifier, at the first turning point described above was first discovered by Yurke et al. [11]. This result was later further developed and extended by Kenig et al. in a series of papers [23], [37], [38], who discovered a similar noise quenching effect at also the second turning point and verified them experimentally. The analyses conducted by both Yurke et al. [11] and Kenig et al. [23], [37], [38] are based on a lumped duffing model of the resonator, whereas our results are based on the hybrid lumped-distributed model. While the results previously obtained based on the lumped model are practically valuable, the distributed model of the beam resonator can offer improved design insight and higher analysis fidelity, and can be used in order to verify the various approximations involved in deriving a lumped model of a distributed component. More complicated resonator structures difficult to model in a lumped manner can be analyzed and optimized for noise performance based on distributed models.

\section{CONCLUSION}

We presented a numerical simulation framework and tool for multidomain systems modeled with coupled PDEs and DAEs. A nontrivial application to an NEMS-based oscillator was discussed. Current and future work is on: 1) compressed, joint Chebyshev-Fourier representations for multivariate functions; 2) full exploitation of structure in hierarchical Chebyshev-Fourier collocation schemes for efficient steady-state and noise analyses for coupled PDE-DAE systems; and 3) in-depth investigation of the noise performance of NEMS feedback oscillators as ultrasensitive sensors.

\section{REFERENCES}

[1] Wikipedia. (2016). Multiphysics-Wikipedia, the Free Encyclopedia. Accessed: Aug. 10, 2016. [Online]. Available: en.wikipedia.org/ w/index.php?title $=$ Multiphysics\&oldid $=727265302$

[2] M. Pedram and S. Nazarian, "Thermal modeling, analysis, and management in VLSI circuits: Principles and methods," Proc. IEEE, vol. 94, no. 8, pp. 1487-1501, Aug. 2006.

[3] K. L. Ekinci and M. L. Roukes, "Nanoelectromechanical systems," Rev. Sci. Instrum., vol. 76, no. 6, 2005, Art. no. 061101.

[4] L. N. Trefethen, Spectral Methods in MATLAB, vol. 10. Philadelphia, PA, USA: SIAM, 2000. 
[5] X. L. Feng, C. J. White, A. Hajimiri, and M. L. Roukes, "A selfsustaining ultrahigh-frequency nanoelectromechanical oscillator," Nat. Nanotechnol., vol. 3, no. 6, pp. 342-346, 2008.

[6] R. Van Leeuwen, D. Karabacak, H. Van der Zant, and W. Venstra, "Nonlinear dynamics of a microelectromechanical oscillator with delayed feedback," Phys. Rev. B, Condens. Matter, vol. 88, no. 21, 2013, Art. no. 214301

[7] K. L. Ekinci, "NEMS: All you need is feedback," Nat. Nanotechnol., vol. 3, no. 6, pp. 319-320, 2008.

[8] M. S. Hanay et al., "Single-protein nanomechanical mass spectrometry in real time," Nat. Nanotechnol., vol. 7, no. 9, pp. 602-608, 2012.

[9] S. Olcum et al., "Suspended nanochannel resonators at attogram precision," in Proc. IEEE 27th Int. Conf. Micro Electro Mech. Syst. (MEMS), San Francisco, CA, USA, 2014, pp. 116-119.

[10] A. N. Cleland and M. L. Roukes, "Noise processes in nanomechanical resonators," J. Appl. Phys., vol. 92, no. 5, pp. 2758-2769, 2002.

[11] B. Yurke, D. S. Greywall, A. N. Pargellis, and P. A. Busch, "Theory of amplifier-noise evasion in an oscillator employing a nonlinear resonator," Phys. Rev. A, vol. 51, no. 5, 1995, pp. 4211-4229.

[12] L. Herrmann, "Vibration of the Euler-Bernoulli beam with allowance for dampings," in Proc. World Congr. Eng., Jul. 2008, pp. 901-904.

[13] V. Novičenko and K. Pyragas, "Phase reduction of weakly perturbed limit cycle oscillations in time-delay systems," Phys. D Nonlin. Phenomena, vol. 241, no. 12, pp. 1090-1098, 2012.

[14] A. Birkisson and T. A. Driscoll, "Automatic Fréchet differentiation for the numerical solution of boundary-value problems," ACM Trans. Math. Softw., vol. 38, no. 4, pp. 1-29, 2012. [Online]. Available: dl.acm.org/citation.cfm?doid=2331130.2331134

[15] G. Floquet, "Sur les équations différentielles linéaires à coefficients périodiques," in Annales scientifiques de l'École normale supérieure, vol. 12. Paris, France: Gauthier-Villars, 1883, pp. 47-88

[16] A. Demir, "Floquet theory and non-linear perturbation analysis for oscillators with differential-algebraic equations," Int. J. Circuit Theory Appl., vol. 28, no. 2, pp. 163-185, Mar./Apr. 2000.

[17] P. A. Kuchment, Floquet Theory for Partial Differential Equations. Berlin, Germany: Birkhäuser, 1993.

[18] Ö. Suvak and A. Demir, "On phase models for oscillators," IEEE Trans. Comput.-Aided Design Integr. Circuits Syst., vol. 30, no. 7, pp. 972-985, Jul. 2011.

[19] The MathWorks, Inc. (2015). MATLAB ${ }^{\circledR}$ Version 2015b. [Online]. Available: http://www.mathworks.com/products/matlab

[20] T. A. Driscoll, N. Hale, and L. N. Trefethen, Chebfun Guide. Oxford, U.K.: Pafnuty, 2014. [Online]. Available: www.chebfun.org/docs/guide/

[21] T. A. Driscoll and N. Hale, "Rectangular spectral collocation," IMA $J$. Numer. Anal., vol. 36, no. 1, pp. 108-132, 2016.

[22] C. T. Kelley, "Arclength continuation and bifurcation," Dept. Math., North Carolina State Univ., Raleigh, NC, USA, Tech. Rep., 2005. [Online]. Available: http://www4.ncsu.edu/ ctk/MA784/continue.pdf

[23] E. Kenig, M. Cross, J. Moehlis, and K. Wiesenfeld, "Phase noise of oscillators with unsaturated amplifiers," Phys. Rev. E, Stat. Phys. Plasmas Fluids Relat. Interdiscipl. Top., vol. 88, no. 6, 2013, Art. no. 062922

[24] L. N. Trefethen, Approximation Theory and Approximation Practice. Philadelphia, PA, USA: SIAM, 2013. [Online]. Available: www2.maths.ox.ac.uk/chebfun/ATAP/

[25] G. B. Wright, M. Javed, H. Montanelli, and L. N. Trefethen, "Extension of Chebfun to periodic functions," SIAM J. Sci. Comput., vol. 37, no. 5, pp. C554-C573, 2015.

[26] A. Townsend and L. N. Trefethen, "An extension of Chebfun to two dimensions," SIAM J. Sci. Comput., vol. 35, no. 6, pp. C495-C518, 2013.

[27] B. Hashemi and L. N. Trefethen. (2016). Chebfun3. Accessed: Jul. 29, 2016. [Online]. Available: www.chebfun.org/docs/guide/guide18.html

[28] B. Hashemi and L. N. Trefethen, "Chebfun in three dimensions," SIAM J. Sci. Comput., vol. 39, no. 9, pp. C341-C363, 2017.

[29] Y. Saad and M. H. Schultz, "GMRES: A generalized minimal residual algorithm for solving nonsymmetric linear systems," SIAM J. Sci. Stat. Comput., vol. 7, no. 3, pp. 856-869, 1986.

[30] O. J. Nastov, "Spectral methods for circuit analysis," Ph.D. dissertation, Dept. Elect. Eng. Comput. Sci., Massachusetts Inst. Technol., Cambridge, MA, USA, 1999.

[31] T. A. Driscoll, "Automatic spectral collocation for integral, integrodifferential, and integrally reformulated differential equations," J. Comput. Phys., vol. 229, no. 17, pp. 5980-5998, 2010.

[32] A. Demir, C. Gu, and J. Roychowdhury, "Phase equations for quasiperiodic oscillators," in Proc. IEEE/ACM Int. Conf. Comput.-Aided Design, San Jose, CA, USA, Nov. 2010, pp. 292-297.
[33] A. Demir, A. Mehrotra, and J. Roychowdhury, "Phase noise in oscillators: A unifying theory and numerical methods for characterization," IEEE Trans. Circuits Syst. I, Fundam. Theory Appl., vol. 47, no. 5, pp. 655-674, May 2000.

[34] A. Demir, "Phase noise and timing jitter in oscillators with colored-noise sources," IEEE Trans. Circuits Syst. I, Fundam. Theory Appl., vol. 49 no. 12, pp. 1782-1791, Dec. 2002.

[35] K. L. Ekinci, Y. T. Yang, and M. L. Roukes, "Ultimate limits to inertial mass sensing based upon nanoelectromechanical systems," J. Appl. Phys., vol. 95, no. 5, pp. 2682-2689, 2004.

[36] M. Sansa et al., "Frequency fluctuations in silicon nanoresonators," Nat. Nanotechnol., vol. 11, no. 6, pp. 552-558, Jun. 2016.

[37] E. Kenig et al., "Optimal operating points of oscillators using nonlinear resonators," Phys. Rev. E, Stat. Phys. Plasmas Fluids Relat. Interdiscip. Top., vol. 86, no. 5, 2012, Art. no. 056207.

[38] L. Villanueva et al., "Surpassing fundamental limits of oscillators using nonlinear resonators," Phys. Rev. Lett., vol. 110, no. 17, 2013, Art. no. 177208.

[39] A. H. Nayfeh and D. T. Mook, Nonlinear Oscillations. New York, NY, USA: Wiley, 1995.

[40] T. Kanamaru, "Duffing oscillator," Scholarpedia, vol. 3, no. 3, p. 6327, 2008.

[41] R. Lifshitz and M. Cross, "Nonlinear dynamics of nanomechanical and micromechanical resonators," in Reviews of Nonlinear Dynamics and Complexity, H. G. Schuster, Ed., vol. 1. Weinheim, Germany: Wiley, 2008, pp. $1-52$.

[42] M. I. McEwan, J. R. Wright, J. E. Cooper, and A. Y. T. Leung, "A combined modal/finite element analysis technique for the dynamic response of a non-linear beam to harmonic excitation," J. Sound Vib., vol. 243, no. 4, pp. 601-624, 2001

[43] B. Yagci, S. Filiz, L. L. Romero, and O. B. Ozdoganlar, "A spectralTchebychev technique for solving linear and nonlinear beam equations," J. Sound Vib., vol. 321, nos. 1-2, pp. 375-404, 2009.

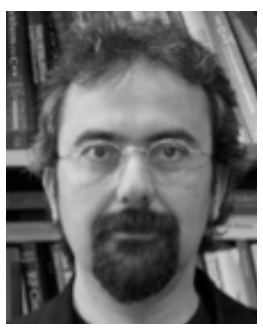

Alper Demir (F'12) received the B.S. degree from Bilkent University, Ankara, Turkey, in 1991, and the M.S. and Ph.D. degrees from the University of California at Berkeley, Berkeley, CA, USA, in 1994 and 1997.

He was with Motorola, Chicago, IL, USA, Cadence Design Systems, San Jose, CA, USA, Bell Laboratories, Murray Hill, NJ, USA, MIT, Cambridge, MA, USA, and University of California at Berkeley. He has been a Faculty Member with Koç University, Istanbul, Turkey, since 2002.

Dr. Demir was a recipient of the 2002 Best of ICCAD Award, the 2003/2014 IEEE/ACM William J. McCalla ICCAD best paper awards, and the 2004 IEEE Guillemin-Cauer Award.

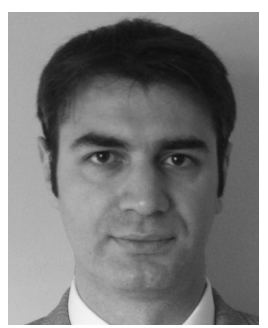

M. Selim Hanay received the B.S. degree from Sabanc1 University, Istanbul, Turkey, in 2003 and the Ph.D. degree from Caltech, Pasadena, CA, USA, in 2011.

He was a Post-Doctoral Research Fellow and a Research Scientist with Caltech. He is currently a Faculty Member with Bilkent University, Ankara, Turkey. His researches on NEMS has been highlighted in the New York Times, IEEE Spectrum, Scientific American, Discover Magazine, and Slashdot.

Dr. Hanay was a recipient of the Gold Medal in the 31st International Chemistry Olympiads. 